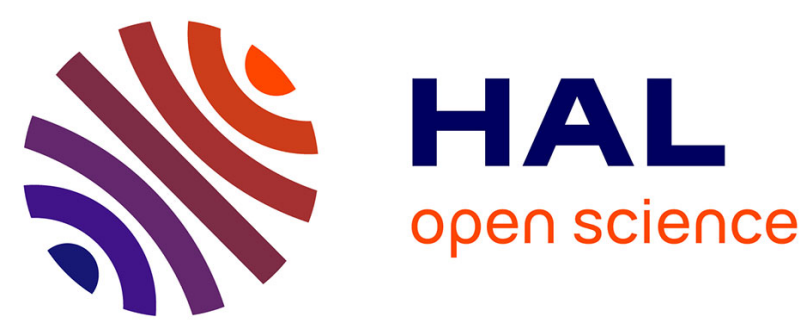

\title{
Investigation of the effective permeability of vuggy or fractured porous media from a Darcy-Brinkman approach
}

\author{
F. Golfier, D. Lasseux, • M Quintard
}

\section{- To cite this version:}

F. Golfier, D. Lasseux, $\bullet$ M Quintard. Investigation of the effective permeability of vuggy or fractured porous media from a Darcy-Brinkman approach. Computational Geosciences, 2015, 19 (1), pp.63-78. 10.1007/s10596-014-9448-5 . hal-03151285

\section{HAL Id: hal-03151285 \\ https://hal.science/hal-03151285}

Submitted on 24 Feb 2021

HAL is a multi-disciplinary open access archive for the deposit and dissemination of scientific research documents, whether they are published or not. The documents may come from teaching and research institutions in France or abroad, or from public or private research centers.
L'archive ouverte pluridisciplinaire HAL, est destinée au dépôt et à la diffusion de documents scientifiques de niveau recherche, publiés ou non, émanant des établissements d'enseignement et de recherche français ou étrangers, des laboratoires publics ou privés. 


\title{
Investigation of the effective permeability of vuggy or fractured porous media from a Darcy-Brinkman approach
}

\author{
F. Golfier · D. Lasseux • M. Quintard
}

\begin{abstract}
In this paper, the macroscopic representation of one-phase incompressible flow in fractured and cavity (or vuggy) porous media is studied from theoretical and numerical points of view. A single-domain (or equivalently a Darcy-Brinkman) type of approach is followed to describe the momentum transport at Darcy scale where the fracture or cavity region and porous matrix region are well identified. The Darcy scale model is upscaled yielding a macroscopic momentum equation operating on the equivalent homogeneous medium. Numerical solution to the associated closure problem is proposed in order to compute the effective permeability. Numerical results on some model fractured and cavity media are discussed and compared to some analytical results.
\end{abstract}

Keywords Vugular rocks · Fractured porous medium . Darcy-Brinkman · Volume averaging · Effective permeability

F. Golfier $(\bowtie)$

GeoRessources Laboratory, Université de Lorraine (ENSG), CNRS, CREGU, F-54501 Vandoeuvre-les-Nancy, France e-mail: fabrice.golfier@ensg.univ-lorraine.fr

\section{Lasseux}

I2M - TREFLE - UMR CNRS 5295, Esplanade des Arts et Métiers, 33405 Talence Cedex, France

e-mail: didier.lasseux@ensam.eu

\section{Quintard}

Université de Toulouse (INPT, UPS) \& CNRS; IMFT (Institut de

Mécanique des Fluides de Toulouse); Allée Camille Soula,

F-31400 Toulouse, France

e-mail: Michel.Quintard@imft.fr

\section{Introduction}

Fractured and vuggy rocks — or cavity media — are a special class of heterogeneous porous media that are of particular interest in petroleum engineering due to the vugular character of some dolomite or limestone oil reservoirs for instance $[8,14,38]$. Such media are characterized by a porous matrix embedding both fractures and macro-poresreferred to as vugs-which characteristic size is several orders of magnitude larger than the matrix pore size. Their typical diameter ranges between $1 \mathrm{~mm}$ and $1 \mathrm{~cm}$ and their presence often results from fossil fragment dissolution. Core analysis on vuggy samples has often exhibited some unexplained behavior like early water breakthrough during imbibition experiments $[12,13]$ and this suggests that a particular attention should be dedicated to macroscopic models and transfer properties of such media while, for instance, vugs distribution within the matrix might play an important role depending on whether they are interconnected through large clusters or totally not connected [31,33, 39, 46-48].

In this paper, the interest is focused on one-phase incompressible creeping flow in vuggy and fractured porous media. A theoretical derivation of a relevant model is discussed first and numerical results on model structures are proposed next.

The situation is schematically depicted in Fig. 1 where we have represented the three different scales of observation of the system under consideration. At the pore-scale, the noncompressible fluid-phase and the solid-phase are identified with their characteristic length-scales $\ell_{\beta}$ and $\ell_{\sigma}$, respectively, while at Darcy's scale, the $\omega$ and $\eta$ regions, referring, respectively, to the porous medium and plain fluid (vugs or fracture) in the rest of this paper, have characteristic lengths $l_{\omega}$ and $l_{\eta}$. At this scale, physical quantities can be obtained by averaging the corresponding pore-scale 


\section{Field (Formation) Scale}

$1 \mathrm{~m}$ to 10 's of $\mathrm{m}$

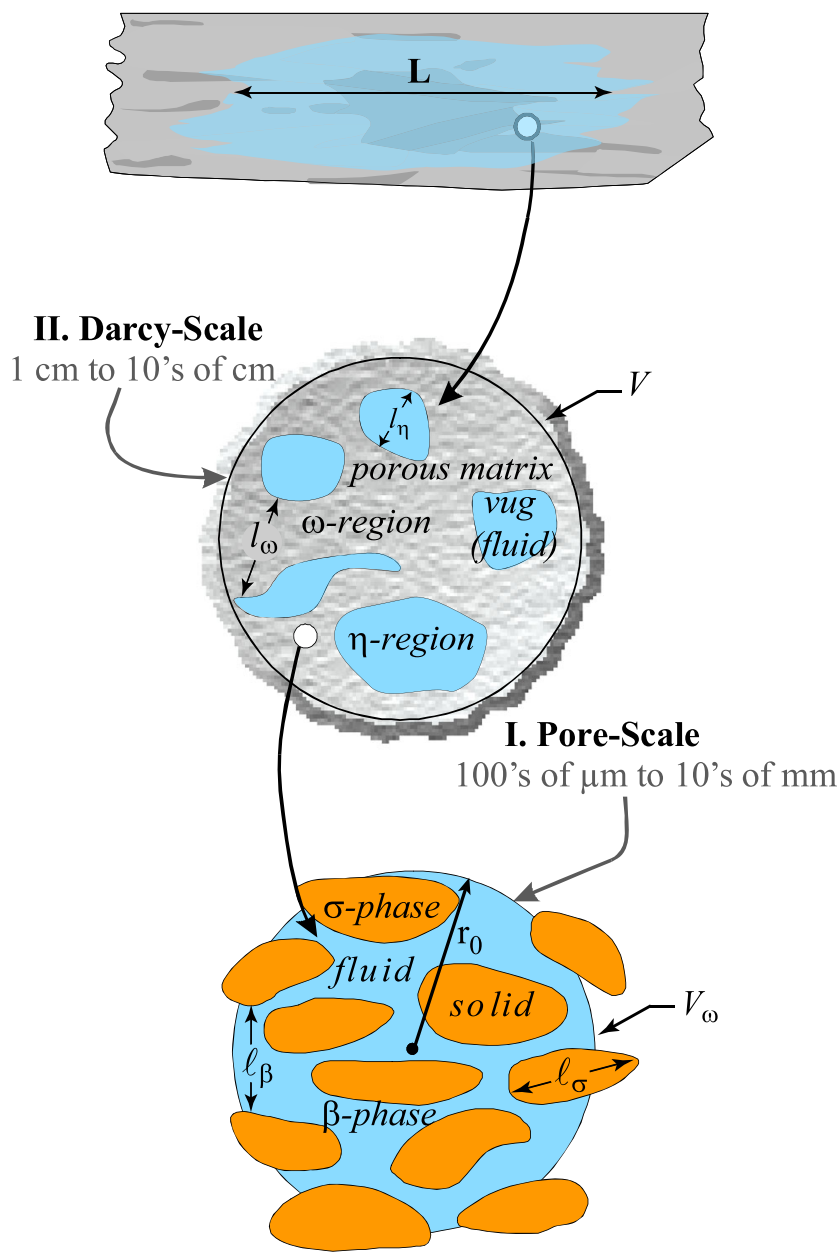

Fig. 1 An example of hierarchy of scales associated with one-phase flow in vuggy rocks

ones over the volume $V_{\omega}$ or $V_{\eta}$. For any physical quantity $\psi_{\beta}$ associated to the $\beta$ phase, it is common to use in both regions the superficial average and the phase intrinsic average, respectively, defined by [64]

$$
\begin{array}{ll}
\left\langle\psi_{\beta}\right\rangle_{\kappa}=\frac{1}{V_{\kappa}} \int_{V_{\beta} \subset V_{\kappa}} \psi_{\beta} d V \quad \kappa=\omega, \eta \\
\left\langle\psi_{\beta}\right\rangle_{\kappa}^{\beta}=\varepsilon_{\kappa}^{-1} \frac{1}{V_{\kappa}} \int_{V_{\beta} \subset V_{\kappa}} \psi_{\beta} d V \quad \kappa=\omega, \eta
\end{array}
$$

with the straightforward relationship

$$
\left\langle\psi_{\beta}\right\rangle_{\kappa}=\varepsilon_{\kappa}\left\langle\psi_{\beta}\right\rangle_{\kappa}^{\beta} \quad \kappa=\omega, \eta
$$

$\varepsilon_{\kappa}$ being the porosity in the $\kappa$-region such as

$$
\varepsilon_{\omega}=\frac{V_{\beta}}{V_{\omega}} \quad \varepsilon_{\eta}=1
$$

For simplication of the notations, the following nomenclature is adopted throughout the article

$$
\begin{array}{ll}
\mathbf{V}_{\beta}^{\kappa}=\left\langle\mathbf{v}_{\beta}\right\rangle_{\kappa} & \kappa=\omega, \eta \\
P_{\beta}^{\kappa}=\left\langle p_{\beta}\right\rangle_{\kappa}^{\beta} & \kappa=\omega, \eta
\end{array}
$$

Finally, the large scale of characteristic length L in Fig. 1 is representative of an equivalent homogeneous structure and the main purpose of our work here is to propose a relevant model over this structure, starting from a description at Darcy's scale in volume $V$.

\section{One- and two-domain approaches}

Situations, for which macroscopic flow equations at the scale $\mathrm{L}$ are required, are encountered in a wide variety of domains and efforts to derive underlying models at Darcy's scale have therefore concentrated a great deal of attention both from theoretical and empirical points of view. The basic approach, sometimes used with success (see, e.g., [43, 49]), consists in extending the validity of Darcy's law, everywhere even in fractures or vugs. An effective permeability value is thus assigned to each inclusion corresponding to the $\eta$-region so that the flow rate passing through the fluid region is recovered. Such a model can be useful if one is not interested in an accurate description of the flow within the fluid domain but it raises, however, the question of the effective permeability value to be used. If, for certain peculiar forms of inclusion (e.g., a two-dimensional fracture of constant thickness), the calculation is straightforward, the question is more tricky in a more general configuration. This issue led to the formulation of various mathematical models of flow in vuggy porous media. A quick overview of the literature following the early works of Brinkman [7], Joseph and Tao [28], and Beavers and Joseph [4] indicates that two main approaches have been followed for the description at the Darcy's scale of one-phase flow in a fluid-porous system to which vugular media are pertaining. These two approaches are sometimes referred to as the single-domain and double-domain approaches.

The first one is based on the use of the Stokes model corrected by a Darcy term in the $\eta$ region (the permeability $K_{\eta}$ being theoretically infinite) and of Darcy's law modified by a Brinkman term in the $\omega$ region of isotropic permeability $K_{\omega}$. It was originally written as

$0=-\nabla P_{\beta}^{\kappa}+\rho_{\beta} \mathbf{g}-\frac{\mu_{\beta}}{K_{\kappa}} \mathbf{V}_{\beta}^{\kappa}+\tilde{\mu} \nabla^{2} \mathbf{V}_{\beta}^{\kappa} \quad(\kappa=\omega, \eta)$

where $\tilde{\mu}=\mu_{\beta}$ in the $\eta$ region. In the $\omega$ region, the correction term $\widetilde{\mu} \nabla^{2} \mathbf{V}_{\beta}^{\omega}$ accounts for the boundary layer type of flow in the neighborhood of the interface with the plain fluid and cancels rapidly beyond a distance of the order of 
$\sqrt{K_{\omega}}$ (or rather, of the grain size [52]) from $A_{\omega \eta}$ inside $\omega$ $[40,57]$. Although confusion has remained on this term, especially on $\tilde{\mu}[34,37]$ as well as on the complete form of the equation-this point will be detailed later on-this model has been employed into many different situations $[2,5,6,19,56]$. As demonstrated in [26], the singledomain model is completely equivalent to the Brinkman approach. This approach makes use of a Darcy-Brinkman equation in the porous region and a Stokes model in the fluid region while the associated boundary conditions are usually assumed to be identical to the case of a fluid/fluid system, i.e., pressure and velocity (or normal stress) continuity. The equivalence between this approach and the single-domain model is exploited in this work.

Conversely, in the double-domain approach, Darcy's law is kept in the porous region and Stokes model in the fluid. From a mathematical point of view, this leads to an ill-posed problem in terms of writing proper boundary conditions to match the two models unless one accepts some discontinuity in the tangential velocity at the interface. Doing so is equivalent to reduce the boundary layer region to a zero thickness surface on which a singularity in the velocity and/or stress fields is concentrated. This was first proposed by Beavers and Joseph $[4,28]$ who postulated a boundary condition of the form

$\frac{d\left(\mathbf{V}_{\beta}^{\eta} \cdot \mathbf{e}_{x}\right)}{d y}=\frac{\alpha}{\sqrt{K_{\omega}}}\left(\mathbf{V}_{\beta}^{\eta}-\mathbf{V}_{\beta}^{\omega}\right) \cdot \mathbf{e}_{x} \quad$ at $A_{\omega \eta}$

in the special case of a two-dimensional channel with a porous wall as represented in Fig. 2i. In Eq. 8, $\alpha$ is a medium-related constant for which no general estimations are available so far. In a study on the slip coefficient values, indeed, Sahraoui and Kaviany [54] showed that it depends on the interfacial location, the permeability, the porosity or surface structure of the porous medium among other parameters. In that sense, $\alpha$ must be considered as an adjustable parameter while the impact remains small and of second order if the interface varies within the range of the grain size [35].

Partial theoretical justifications of this approach were proposed $[9,15,25,53]$ and were used practically in the (i)

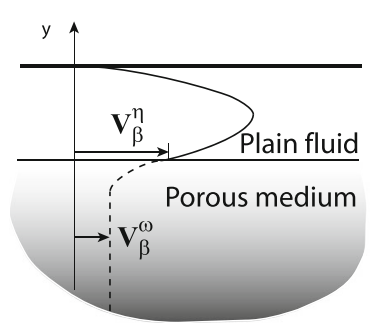

(ii)

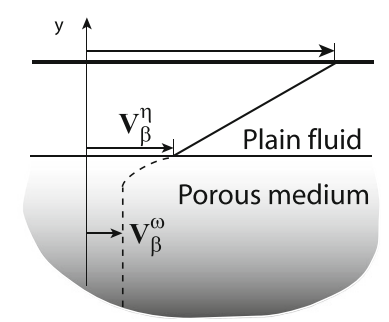

Fig. 2 Geometry with Poiseuille flow considered in [4] and [40] (i); geometry with Couette flow considered in [29] (ii) case of the simple geometry represented in Fig. 2i. For this specific configuration, it was demonstrated that the singleand double-domain approaches are identical and lead to the same result for the flow within the fluid region only, provided $\alpha$ is identified to $\sqrt{\frac{\widetilde{\mu}}{\mu_{\beta}}}$ [40]. In the case of a shear flow past a porous body (Fig. 2ii), the analogy requires $\alpha=\frac{\widetilde{\mu}}{\mu_{\beta}}$ as shown in [29]. Extensions or modifications of this interfacial condition were proposed by Saffman [53] and Jones [27] in order to consider more complex geometries.

More recently, the problem was carefully revisited from a theoretical point of view in an original analysis developed with the aid of the volume averaging method [44]. Under the classical constraint that scales are well separated, it was shown that two corrective terms-referred to as the first and second Brinkman corrections-both of the same order of magnitude in the $\omega-\eta$ interfacial region, must be considered to describe the flow with a unique momentum equation in the entire $\omega$ region. This equation can be written under the form

$$
\begin{aligned}
0= & -\nabla P_{\beta}^{\omega}+\rho_{\beta} \mathbf{g}+\frac{\mu_{\beta}}{\varepsilon_{\omega}} \nabla^{2} \mathbf{V}_{\beta}^{\omega} \\
& -\frac{\mu_{\beta}}{\varepsilon_{\omega}} \nabla \varepsilon_{\omega} \cdot \nabla\left(\varepsilon_{\beta}^{-1} \mathbf{V}_{\beta}^{\omega}\right)-\mu_{\beta} \Phi_{\beta}
\end{aligned}
$$

The first Brinkman correction term corresponds to a viscous diffusive term (see Eq. 7) while the second one takes into account, through the porosity gradient, the modification of the structure in $\omega$ near the interface with the fluid ( $\eta$-region). An important feature is that the viscosity associated to the diffusive term in Eq. 9 is actually $\tilde{\mu}=\frac{\mu_{\beta}}{\varepsilon_{\omega}}$ as already proved in previous works [63]. In the $\eta$-region, and provided some lengthscale constraints are fulfilled, the averaged equations were justified to be formally the same as the point equations, and in particular, the momentum balance is given by

$0=-\nabla P_{\beta}^{\eta}+\rho_{\beta} \mathbf{g}+\mu_{\beta} \nabla^{2} \mathbf{V}_{\beta}^{\eta}$

Unfortunately, Eq. 9 is not a closed form since an expression for $\Phi_{\beta}$ independent of non-local pressure and velocity (i.e., integro-differential terms) and valid everywhere in the $\omega$-region cannot be found. A local closed form is available in the region far from the interface only, i.e., in the homogeneous part of $\omega$, where it can be shown that $\Phi_{\beta}$ is given by $\mathbf{K}_{\omega}^{-1} \cdot\left\langle\mathbf{v}_{\beta}\right\rangle_{\omega}$ and Eq. 9 can be written as

$0=-\nabla P_{\beta}^{\omega}+\rho_{\beta} \mathbf{g}+\frac{\mu_{\beta}}{\varepsilon_{\omega}} \nabla^{2} \mathbf{V}_{\beta}^{\omega}-\mu_{\beta} \mathbf{K}_{\omega}^{-1} \cdot \mathbf{V}_{\beta}^{\omega}$

Because of this difficulty, Ochoa-Tapia and Whitaker further proposed to use Eq. 11 everywhere in the $\omega$ region and to compensate the inaccuracy close to $A_{\omega \eta}$, they developed a jump condition at the $\omega-\eta$ boundary for this equation to be valid everywhere in $\omega$ on an average sense. In that way, as in the Beavers-Joseph type of approach, a particular physics is attributed to the interface. This jump condition 
was rigorously justified in [45] for the one-dimensional tangential flow sketched in Fig. 2. Keeping the velocity field continuous at the interface, i.e.,

$\mathbf{V}_{\beta}^{\omega}=\mathbf{V}_{\beta}^{\eta} \quad$ at $A_{\omega \eta}$

the excess stress can be expressed under the following generalized form

$$
\begin{aligned}
\mathbf{n}_{\omega \eta} \cdot & {\left[\left(P_{\beta}^{\eta}-P_{\beta}^{\omega}\right) \mathbf{I}+\mu_{\beta}\left[\left(\varepsilon_{\omega}^{-1} \nabla \mathbf{V}_{\beta}^{\omega}-\nabla \mathbf{V}_{\beta}^{\eta}\right)\right.\right.} \\
+ & \left.\left.\left(\varepsilon_{\omega}^{-1} \nabla \mathbf{V}_{\beta}^{\omega}-\nabla \mathbf{V}_{\beta}^{\eta}\right)^{T}\right)\right]=\mu_{\beta} \mathbf{M} \cdot \mathbf{V}_{\beta}^{\omega} \text { at } A_{\omega \eta}
\end{aligned}
$$

In this relationship, $\mathbf{M}$ is a viscous drag tensor depending on $\mathbf{K}_{\omega}$ and $\varepsilon_{\omega}$ and must be considered as an adjustable parameter. Note that, in the problem studied in [45], the contribution of the transpose term is zero when the flow is parallel to the dividing surface. A quick order of magnitude analysis would indicate that

$\mathbf{M}=O\left(K_{\omega}^{-\frac{1}{2}}\right)$

which is in agreement with the fact that the flow structure exhibits significant variation over a distance of the order of $\sqrt{K_{\omega}}$ from the interface. In the case of a simple configuration with forced flow parallel to the interface-similar to the one investigated in [4] (Fig. 2i)—Goyeau et al. [20] developed an explicit relation for this viscous drag tensor (that reduces to a scalar jump coefficient in that case). Recently, this work was pursued [62] leading to the derivation of local closure problems governing this stress jump at the interfacial region but still for the same simple configuration. A novel and more sophisticated methodology was proposed in [60] for the derivation of these jump boundary conditions. Numerical investigations were also carried out $[3,26,58,61]$ but again in the simple configuration envisaged by Beavers and Joseph. Some results in a more general situation are available in [30].

Clearly, the question of which theory is the most convenient one for describing flow in a plain fluid-porous medium system is still a subject of controversy (see, e.g., [41, 42, 59]). In the general case of cavity media where size and spatial distribution of the $\omega$ and $\eta$ regions can be complex and for which the flow pattern is much more complicated than the simple parallel flow usually investigated, the question is even more open. However, as shown in [58], significant mismatch between the different approaches is expected for large enough values of $\varepsilon_{\omega}$ and $K_{\omega}$ only. Numerical results in [58] show no difference in the flow field obtained with a jump boundary condition (Eq. 13) or with the classical Brinkman approach when $\varepsilon_{\omega}<0.6$ and $K_{\omega}<3 D$. This is further confirmed by the work reported in [30]. In this reference, macroscopic numerical results obtained on several different configurations and sets of parameters did not show very significant differences between the two approaches in comparison with corresponding averaged microscale results. While a long-term objective would be to derive a nonlocal closed form of Eq. 9 in the general case, the investigation of the present work is focused on the possibility of describing vuggy or fractured media by a large-scale effective permeability and to provide an explicit way of determining this coefficient for a given matrix and vug structure. This goes along the same lines as a recent work reported by Arbogast and Lehr [1] using homogenization theory and a Beavers and Joseph doubledomain approach. More recently, this study was extended to more general fractured vuggy systems using either a DarcyBrinkman (one-domain) approach $[32,50]$ or a Beavers and Joseph formulation [24]. In this last reference, numerical simulations were carried out on vugular porous structures at two different scales. At the Darcy-scale, the incompressible Darcy-Brinkman flow (one-domain approach) was solved on the structure using a fine grid discretization. At the large-scale, the Darcy equation, obtained by upscaling the Darcy-Brinkman model, was solved using a coarse grid discretization, each grid-block bearing an effective permeability computed from the average over each block of the flow solution at the fine-grid description. Results at both scales were satisfactorily compared. The net achievement of this work was the validation of the upscaling procedure.

While keeping the same Darcy-scale model, our objective in the present work is somewhat different. First, we carefully derive the large-scale model that is validated using an analytical solution on some model structure. Comparison of this Darcy-Brinkman approach is then carried out on the same structure with a two-domain (Beavers-Joseph) approach in a second step.

The starting point of our analysis is a Brinkman type of description at Darcy scale for one-phase incompressible flow in a vugular or fractured media, i.e., a Darcy-Brinkman equation in the $\omega$-region and Stokes flow in the $\eta$-region with an interface $A_{\omega \eta}$ separating the two on which velocity and stress continuity boundary conditions are applied. This description results from a first upscaling performed on the Stokes model at the pore-scale as presented in [33] using the averaging volume $V_{\eta}$ either in the $\eta$-region containing the fluid phase $\beta$ only or $V_{\omega}$ in the $\omega$-region containing both the solid rigid phase $\sigma$ and the $\beta$ phase. The model is chosen based on the idea that (i) it must account for any flow pattern whatever the distribution and shapes of matrix and vugs; (ii) the ensuing macroscopic model is expected to be predictive without adjustable parameters; (iii) Darcy scale properties in the $\omega$-region do not evolve significantly over a distance from $A_{\omega \eta}$ larger than $\sqrt{K_{\omega}}$ or grain size of the matrix. The macroscopic model operating at scale $\mathrm{L}$ is obtained from a second up-scaling that makes use of the averaging volume $V$ (Fig. 1) where matrix and vugs are clearly identified with their characteristic length scale 
$l_{\omega}$ and $l_{\eta}$, the final macroscopic structure having a generic length $\mathrm{L}$. The main outcome of the work resulting from this procedure is a large-scale momentum conservation equation formally identical to a Darcy equation that involves an effective permeability entirely defined by the associated closure. Illustrative results obtained from numerical solutions of the closure problem over simple unit cells representative of a model fractured medium and vugular porous structure are provided and commented through comparisons with existing works.

\section{Volume averaging}

We start the derivation of the macroscopic model by recalling the averaged form of the mass and momentum balance equations in the two regions that can be written as [44]

$\nabla \cdot \mathbf{V}_{\beta}^{\omega}=0$

$0=-\nabla P_{\beta}^{\omega}+\rho_{\beta} \mathbf{g}+\frac{\mu_{\beta}}{\varepsilon_{\omega}} \nabla^{2} \mathbf{V}_{\beta}^{\omega}-\mu_{\beta} \mathbf{K}_{\omega}^{-1} \cdot \mathbf{V}_{\beta}^{\omega}$

in the $\omega$-region

$\nabla \cdot \mathbf{V}_{\beta}^{\eta}=0$

$0=-\nabla P_{\beta}^{\eta}+\rho_{\beta} \mathbf{g}+\mu_{\beta} \nabla^{2} \mathbf{V}_{\beta}^{\eta} \quad$ in the $\eta$-region

To arrive at this result, one must specify that the radius $r_{0}$ (Fig. 1) of the averaging volume $V_{\kappa}(\kappa=\omega, \eta)$ satisfies the following length-scale constraints [64]

$r_{0} \gg l_{\beta}, r_{0}^{2} \ll L_{\varepsilon} L_{p 1}, r_{0}^{2} \ll L_{v} L_{v 1}, r_{0}^{2} \ll L_{\varepsilon} L_{v 2}$

In this scales hierarchy, the respective length-scales $L_{\varepsilon}$, $L_{p 1}, L_{v}, L_{v 1}$, and $L_{v 2}$ are defined as the distances over which $\varepsilon, \nabla p_{\beta}, \mathbf{v}_{\beta}, \nabla \mathbf{v}_{\beta}$, and $\nabla \nabla \mathbf{v}_{\beta}$ are experiencing significant changes with respect to an a priori fixed criterion. Here, $\varepsilon$ is considered as a constant and, due to the heterogeneous structure with characteristic lengths $l_{\eta}$ and $l_{\omega}$, the three last constraints in Eq. 19 can be replaced by

$r_{0} \ll l_{\eta}, \quad r_{0} \ll l_{\omega}$

As detailed in the introduction, this description-coupled with velocity and stress continuity boundary conditionsis formally equivalent to the single-domain approach where the Darcy-Brinkman equation is valid everywhere (i.e., with $\varepsilon_{\eta}=1$ and $\left.K_{\eta} \rightarrow \infty\right)$. Since no jump is assumed on $A_{\omega \eta}$, it can be easily shown that the boundary conditions associated to the above balance equations are

$\mathbf{V}_{\beta}^{\omega}=\mathbf{V}_{\beta}^{\eta} \quad$ at $A_{\omega \eta}$

$$
\begin{aligned}
\mathbf{n}_{\omega \eta} \cdot\left[\left(P_{\beta}^{\eta}\right.\right. & \left.-P_{\beta}^{\omega}\right) \mathbf{I}+\mu_{\beta}\left[\left(\varepsilon_{\omega}^{-1} \nabla \mathbf{V}_{\beta}^{\omega}-\nabla \mathbf{V}_{\beta}^{\eta}\right)\right. \\
& \left.\left.+\left(\varepsilon_{\omega}^{-1} \nabla \mathbf{V}_{\beta}^{\omega}-\nabla \mathbf{V}_{\beta}^{\eta}\right)^{T}\right)\right]=0 \quad \text { at } A_{\omega \eta}
\end{aligned}
$$

We now proceed with the development of the macroscopic model by averaging Eqs. 16-18 and 21-22 using the volume $V$ (see Fig. 1). We refer the reader to the cited literature for a complete overview of the volume averaging technique, and the associated developments.

In the following, we use the notations for the large-scale averages:

$$
\begin{aligned}
& \left\{\Psi_{\beta}^{\kappa}\right\}_{\kappa}=\frac{1}{V} \int_{V_{\kappa}} \Psi_{\beta}^{\kappa} d V \quad(\kappa=\omega, \eta) \\
& \Psi_{\beta}^{*}=\left\{\Psi_{\beta}\right\}=\frac{1}{V} \int_{V} \Psi_{\beta} d V=\left\{\Psi_{\beta}^{\omega}\right\}_{\omega}+\left\{\Psi_{\beta}^{\eta}\right\}_{\eta}
\end{aligned}
$$

\subsection{Mass balance equation}

As for the pore-scale problem, the superficial average of the mass conservation equation, Eq. 15, can be formed to obtain

$$
\begin{aligned}
\left\{\nabla \cdot \mathbf{V}_{\beta}^{\omega}\right\}_{\omega} & =\nabla \cdot\left(\frac{1}{V} \int_{V_{\omega}} \mathbf{V}_{\beta}^{\omega} d V\right)+\frac{1}{V} \int_{\mathcal{A}_{\omega \eta}} \mathbf{n}_{\omega \eta} \cdot \mathbf{V}_{\beta}^{\omega} d A \\
& =0
\end{aligned}
$$

and this is achieved by making use of the volume averaging theorem [23]

$$
\left\{\nabla \Psi_{\beta}^{\kappa}\right\}_{\kappa}=\nabla\left\{\Psi_{\beta}^{\kappa}\right\}_{\kappa}+\frac{1}{V} \int_{\mathcal{A}_{\omega \eta}} \mathbf{n}_{\omega \eta} \Psi_{\beta}^{\kappa} d A
$$

The same procedure can be used with Eq. 17 to obtain

$$
\begin{aligned}
\left\{\nabla \cdot \mathbf{V}_{\beta}^{\eta}\right\}_{\eta} & =\nabla \cdot\left(\frac{1}{V} \int_{V_{\eta}} \mathbf{V}_{\beta}^{\eta} d V\right)+\frac{1}{V} \int_{\mathcal{A}_{\omega \eta}} \mathbf{n}_{\eta \omega} \cdot \mathbf{V}_{\beta}^{\eta} d A \\
& =0
\end{aligned}
$$

If Eqs. 25 and 27 are added, one readily finds:

$$
\begin{array}{r}
\nabla \cdot\left(\frac{1}{V} \int_{V_{\omega}} \mathbf{V}_{\beta}^{\omega} d V+\frac{1}{V} \int_{V_{\eta}} \mathbf{V}_{\beta}^{\eta} d V\right) \\
+\frac{1}{V} \int_{\mathcal{A}_{\omega \eta}}\left(\mathbf{n}_{\omega \eta} \cdot \mathbf{V}_{\beta}^{\omega}+\mathbf{n}_{\eta \omega} \cdot \mathbf{V}_{\beta}^{\eta}\right) d A=0
\end{array}
$$

and if the boundary condition, Eq. 21, is used with the fact that

$\mathbf{n}_{\omega \eta}=-\mathbf{n}_{\eta \omega} \quad$ on $A_{\omega \eta}$ 
Equation 28 then writes:

$\nabla \cdot \mathbf{V}_{\beta}^{*}=0$

\subsection{Momentum balance equation}

For the momentum balance equations, Eqs. 16 and 18, one can easily arrive at

$$
\begin{gathered}
0=-\nabla\left\{P_{\beta}^{\omega}\right\}_{\omega}-\frac{1}{V} \int_{\mathcal{A}_{\omega \eta}} \mathbf{n}_{\omega \eta} P_{\beta}^{\omega} d A+\rho_{\beta} \mathbf{g} \phi_{\omega} \\
+\frac{\mu_{\beta}}{\varepsilon_{\omega}} \nabla \cdot\left\{\nabla \mathbf{V}_{\beta}^{\omega}\right\}_{\omega}-\mu_{\beta}\left\{\mathbf{K}_{\omega}^{-1} \cdot \mathbf{V}_{\beta}^{\omega}\right\}_{\omega} \\
+\frac{\mu_{\beta}}{\varepsilon_{\omega}} \frac{1}{V} \int_{\mathcal{A}_{\omega \eta}} \mathbf{n}_{\omega \eta} \cdot\left(\nabla \mathbf{V}_{\beta}^{\omega}+\nabla \mathbf{V}_{\beta}^{\omega T}\right) d A
\end{gathered}
$$

and

$$
\begin{aligned}
& 0=-\nabla\left\{P_{\beta}^{\eta}\right\}_{\eta}-\frac{1}{V} \int_{\mathcal{A}_{\omega \eta}} \mathbf{n}_{\eta \omega} P_{\beta}^{\eta} d A+\rho_{\beta} \mathbf{g} \phi_{\eta} \\
& +\mu_{\beta} \nabla \cdot\left\{\nabla \mathbf{V}_{\beta}^{\eta}\right\}_{\eta} \\
& \quad+\mu_{\beta} \frac{1}{V} \int_{\mathcal{A}_{\omega \eta}} \mathbf{n}_{\eta \omega} \cdot\left(\nabla \mathbf{V}_{\beta}^{\eta}+\nabla \mathbf{V}_{\beta}^{\eta T}\right) d A
\end{aligned}
$$

Here, we have used the fact that, due to the mass balance Eqs. 15 and $17, \nabla \cdot \nabla \mathbf{V}_{\beta}^{\omega T}=0$ and $\nabla \cdot \nabla \mathbf{V}_{\beta}^{\eta T}=0$.

Summing up both equations leads to

$$
\begin{aligned}
& 0=-\nabla P_{\beta}^{*}-\frac{1}{V} \int_{\mathcal{A}_{\omega \eta}} \mathbf{n}_{\omega \eta}\left(P_{\beta}^{\omega}-P_{\beta}^{\eta}\right) d A+\rho_{\beta} \mathbf{g} \\
& +\frac{\mu_{\beta}}{\varepsilon_{\omega}} \nabla \cdot\left\{\nabla \mathbf{V}_{\beta}^{\omega}\right\}_{\omega}+\mu_{\beta} \nabla \cdot\left\{\nabla \mathbf{V}_{\beta}^{\eta}\right\}_{\eta} \\
& +\mu_{\beta} \frac{1}{V} \int_{\mathcal{A}_{\omega \eta}} \mathbf{n}_{\omega \eta} \cdot\left(\frac{1}{\varepsilon_{\omega}} \nabla \mathbf{V}_{\beta}^{\omega}-\nabla \mathbf{V}_{\beta}^{\eta}\right) d A \\
& +\mu_{\beta} \frac{1}{V} \int_{\mathcal{A}_{\omega \eta}} \mathbf{n}_{\omega \eta} \cdot\left(\frac{1}{\varepsilon_{\omega}} \nabla \mathbf{V}_{\beta}^{\omega}-\nabla \mathbf{V}_{\beta}^{\eta}\right)^{T} d A \\
& \quad-\mu_{\beta}\left\{\mathbf{K}_{\omega}^{-1} \cdot \mathbf{V}_{\beta}^{\omega}\right\}_{\omega}
\end{aligned}
$$

From the boundary condition on $\mathcal{A}_{\omega \eta}$, Eq. 22, we write

$$
\begin{array}{r}
\frac{1}{V} \int_{\mathcal{A}_{\omega \eta}} \mathbf{n}_{\omega \eta} \cdot\left[\left(P_{\beta}^{\eta}-P_{\beta}^{\omega}\right) \mathbf{I}+\mu_{\beta}\left(\varepsilon_{\omega}^{-1} \nabla \mathbf{V}_{\beta}^{\omega}-\nabla \mathbf{V}_{\beta}^{\eta}\right)\right. \\
\left.+\mu_{\beta}\left(\varepsilon_{\omega}^{-1} \nabla \mathbf{V}_{\beta}^{\omega}-\nabla \mathbf{V}_{\beta}^{\eta}\right)^{T}\right] d A=0
\end{array}
$$

which leads to the following averaged form of the DarcyBrinkman equation

$$
\begin{aligned}
0=-\nabla P_{\beta}^{*}+ & \rho_{\beta} \mathbf{g}+\frac{\mu_{\beta}}{\varepsilon_{\omega}} \nabla \cdot\left\{\nabla \mathbf{V}_{\beta}^{\omega}\right\}_{\omega} \\
& +\mu_{\beta} \nabla \cdot\left\{\nabla \mathbf{V}_{\beta}^{\eta}\right\}_{\eta}-\mu_{\beta}\left\{\mathbf{K}_{\omega}^{-1} \cdot \mathbf{V}_{\beta}^{\omega}\right\}_{\omega}
\end{aligned}
$$

Substituting decompositions for local velocity and pressure [21]

$P_{\beta}^{\kappa}=P_{\beta}^{*}+\widetilde{P}_{\beta}^{\kappa} \quad \kappa=\omega, \eta$

$\mathbf{V}_{\beta}^{\kappa}=\mathbf{V}_{\beta}^{*}+\widetilde{\mathbf{V}}_{\beta}^{\kappa} \quad \kappa=\omega, \eta$

where the quantities $\widetilde{P}_{\beta}^{\kappa}$ and $\widetilde{\mathbf{V}}_{\beta}^{\kappa}$ are referred to as spatial deviation terms, we get

$$
\begin{aligned}
& 0=-\nabla P_{\beta}^{*}+\rho_{\beta} \mathbf{g}+\mu_{\beta}\left(\frac{\phi_{\omega}}{\varepsilon_{\omega}}+\phi_{\eta}\right) \nabla^{2} \mathbf{V}_{\beta}^{*} \\
& +\frac{\mu_{\beta}}{\varepsilon_{\omega}} \nabla \cdot\left\{\nabla \tilde{\mathbf{V}}_{\beta}^{\omega}\right\}_{\omega}+\mu_{\beta} \nabla \cdot\left\{\nabla \tilde{\mathbf{V}}_{\beta}^{\eta}\right\}_{\eta} \\
& \quad-\mu_{\beta} \phi_{\omega} \mathbf{K}_{\omega}^{-1} \cdot \mathbf{V}_{\beta}^{*}-\mu_{\beta} \mathbf{K}_{\omega}^{-1} \cdot\left\{\tilde{\mathbf{V}}_{\beta}^{\omega}\right\}_{\omega}
\end{aligned}
$$

Here, $\mathbf{V}_{\beta}^{*}$ and $\nabla \mathbf{V}_{\beta}^{*}$ are considered as constant over the integration volume $V$.

\subsection{Closure}

At this point, a way of closing our problem is to set up the governing equations of the deviation fields. Subtracting unclosed equations, Eqs. 30 and 38, from the set of pore-scale equations, Eqs. 15-18, leads to the following relationships

Deviation equations in the $\omega$-region

$\nabla \cdot \tilde{\mathbf{V}}_{\beta}^{\omega}=0$

$$
\begin{aligned}
& 0=-\nabla \widetilde{P}_{\beta}^{\omega}+\mu_{\beta} \phi_{\eta}\left(\frac{1}{\varepsilon_{\omega}}-1\right) \nabla^{2} \mathbf{V}_{\beta}^{*}+\frac{\mu_{\beta}}{\varepsilon_{\omega}} \nabla^{2} \widetilde{\mathbf{V}}_{\beta}^{\omega} \\
& -\frac{\mu_{\beta}}{\varepsilon_{\omega}} \nabla \cdot\left\{\nabla \widetilde{\mathbf{V}}_{\beta}^{\omega}\right\}_{\omega}-\mu_{\beta} \nabla \cdot\left\{\nabla \widetilde{\mathbf{V}}_{\beta}^{\eta}\right\}_{\eta} \\
& -\mu_{\beta} \phi_{\eta} \mathbf{K}_{\omega}^{-1} \cdot \mathbf{V}_{\beta}^{*}-\mu_{\beta} \mathbf{K}_{\omega}^{-1} \cdot \widetilde{\mathbf{V}}_{\beta}^{\omega}+\mu_{\beta} \mathbf{K}_{\omega}^{-1} \cdot\left\{\widetilde{\mathbf{V}}_{\beta}^{\omega}\right\}_{\omega}
\end{aligned}
$$

\section{Deviation equations in the $\eta$-region}

$\nabla \cdot \widetilde{\mathbf{v}}_{\beta}^{\eta}=0$

$$
\begin{gathered}
0=-\nabla \widetilde{P}_{\beta}^{\eta}-\mu_{\beta} \phi_{\omega}\left(\frac{1}{\varepsilon_{\omega}}-1\right) \nabla^{2} \mathbf{V}_{\beta}^{*}+\mu_{\beta} \nabla^{2} \widetilde{\mathbf{V}}_{\beta}^{\eta} \\
-\frac{\mu_{\beta}}{\varepsilon_{\omega}} \nabla \cdot\left\{\nabla \widetilde{\mathbf{V}}_{\beta}^{\omega}\right\}_{\omega}-\mu_{\beta} \nabla \cdot\left\{\nabla \widetilde{\mathbf{V}}_{\beta}^{\eta}\right\}_{\eta} \\
+\mu_{\beta} \phi_{\omega} \mathbf{K}_{\omega}^{-1} \cdot \mathbf{V}_{\beta}^{*}+\mu_{\beta} \mathbf{K}_{\omega}^{-1} \cdot\left\{\widetilde{\mathbf{V}}_{\beta}^{\omega}\right\}_{\omega}
\end{gathered}
$$


At this point, simplifications can be made in the momentumlike equations for the deviations on the basis of an order of magnitude analysis carried out on the various terms while keeping in mind the constraint of separation of scales,

$l_{\omega}, l_{\eta} \ll \mathrm{L}$

First, the definition of $\mathbf{V}_{\beta}^{*}$ allows the straightforward following order of magnitude estimate

$\mathbf{V}_{\beta}^{*}=\mathcal{O}\left(\phi_{\eta} \tilde{\mathbf{V}}_{\beta}^{\eta}+\phi_{\omega} \tilde{\mathbf{V}}_{\beta}^{\omega}\right)$

from which we can derive

$\phi_{\eta} \tilde{\mathbf{V}}_{\beta}^{\eta}=\mathcal{O}\left(\mathbf{V}_{\beta}^{*}\right) \quad ; \quad \phi_{\omega} \tilde{\mathbf{V}}_{\beta}^{\omega}=\mathcal{O}\left(\mathbf{V}_{\beta}^{*}\right)$

with the idea that these two last relationships represent upper bound estimates for $\widetilde{\mathbf{V}}_{\beta}^{\eta}$ and $\widetilde{\mathbf{V}}_{\beta}^{\omega}$. As a consequence, an upper bound for the estimate of the Brinkman-like terms in the right-hand side of Eq. 40 is

$$
\begin{aligned}
& \frac{\mu_{\beta}}{\varepsilon_{\omega}} \nabla \cdot\left\{\nabla \tilde{\mathbf{V}}_{\beta}^{\omega}\right\}_{\omega}+\mu_{\beta} \nabla \cdot\left\{\nabla \tilde{\mathbf{V}}_{\beta}^{\eta}\right\}_{\eta}= \\
& \mathcal{O}\left(\frac{\mu_{\beta}}{\mathrm{L}}\left(\frac{1}{\varepsilon_{\omega} l_{\omega}}+\frac{1}{l_{\eta}}\right) \mathbf{V}_{\beta}^{*}\right)
\end{aligned}
$$

This clearly indicates that these two Brinkman-like terms are fully negligible compared to the Darcy term $\mu_{\beta} \phi_{\eta} \mathbf{K}_{\omega}^{-1}$. $\mathbf{V}_{\beta}^{*}$ and this is due to the fact that, in any practical situation,

$\mathcal{O}\left(\frac{1}{\mathrm{~L}}\left(\frac{1}{\varepsilon_{\omega} l_{\omega}}+\frac{1}{l_{\eta}}\right)\right) \ll \mathcal{O}\left(\phi_{\eta} \mathbf{K}_{\omega}^{-1}\right)$

Similarly, the first Brinkman-like term in the right-hand side of Eq. 40 is such that

$\mu_{\beta} \phi_{\eta}\left(\frac{1}{\varepsilon_{\omega}}-1\right) \underbrace{\nabla^{2} \mathbf{V}_{\beta}^{*}}_{\frac{\mathbf{V}_{\beta}^{*}}{\mathrm{~L}^{2}}} \ll \mu_{\beta} \phi_{\eta} \underbrace{\mathbf{K}_{\omega}^{-1} \cdot \mathbf{V}_{\beta}^{*}}_{\mathbf{K}_{\omega}^{-1} \cdot \mathbf{V}_{\beta}^{*}}$

due to the fact that, in all real configurations,

$\mathcal{O}\left(\mathbf{K}_{\omega}\right) \ll \mathcal{O}\left(\frac{\mathrm{L}^{2}}{\frac{1}{\varepsilon_{\omega}}-1}\right)$

Similar relations hold for Eq. 42 so that the pore-scale boundary value problem for the deviation equations takes the following form

Deviation equations in the $\omega$-region

$\nabla \cdot \tilde{\mathbf{V}}_{\beta}^{\omega}=0$

$$
\begin{aligned}
0= & -\nabla \widetilde{P}_{\beta}^{\omega}+\frac{\mu_{\beta}}{\varepsilon_{\omega}} \nabla^{2} \tilde{\mathbf{V}}_{\beta}^{\omega}-\mu_{\beta} \phi_{\eta} \mathbf{K}_{\omega}^{-1} \cdot \mathbf{V}_{\beta}^{*} \\
& -\mu_{\beta} \mathbf{K}_{\omega}^{-1} \cdot \tilde{\mathbf{V}}_{\beta}^{\omega}+\mu_{\beta} \mathbf{K}_{\omega}^{-1} \cdot\left\{\tilde{\mathbf{V}}_{\beta}^{\omega}\right\}_{\omega}
\end{aligned}
$$

\section{Boundary conditions}

B.C. $1 \quad \tilde{\mathbf{V}}_{\beta}^{\omega}=\tilde{\mathbf{V}}_{\beta}^{\eta} \quad$ at $A_{\omega \eta}$

$$
\begin{aligned}
& \text { B.C. } 2 \quad \mathbf{n}_{\omega \eta} \cdot\left(\left(\widetilde{P}_{\beta}^{\eta}-\widetilde{P}_{\beta}^{\omega}\right) \mathbf{I}\right. \\
& \begin{aligned}
\left.+\mu_{\beta}\left(\frac{1}{\varepsilon_{\omega}} \nabla \widetilde{\mathbf{V}}_{\beta}^{\omega}-\nabla \widetilde{\mathbf{V}}_{\beta}^{\eta}\right)+\mu_{\beta}\left(\frac{1}{\varepsilon_{\omega}} \nabla \widetilde{\mathbf{V}}_{\beta}^{\omega}-\nabla \widetilde{\mathbf{V}}_{\beta}^{\eta}\right)^{T}\right) \\
=0 \text { at } A_{\omega \eta}
\end{aligned}
\end{aligned}
$$

In this last boundary condition, we have omitted the macro-scale term $\mu_{\beta}\left(\frac{1}{\varepsilon_{\omega}}-1\right) \mathbf{n}_{\omega \eta} \cdot\left(\nabla \mathbf{V}_{\beta}^{*}+\nabla \mathbf{V}_{\beta}^{* T}\right)$ since this term is completely negligible compared to the deviation terms of the viscous shear. This straightforwardly follows from the order of magnitude estimate of $\mathbf{V}_{\beta}^{*}$ given in Eq. 44 and scale hierarchy expressed in Eq. 43.

Deviation equations in the $\eta$-region

$\nabla \cdot \tilde{\mathbf{V}}_{\beta}^{\eta}=0$

$$
\begin{aligned}
0= & -\nabla \widetilde{P}_{\beta}^{\eta}+\mu_{\beta} \nabla^{2} \widetilde{\mathbf{V}}_{\beta}^{\eta} \\
& +\mu_{\beta} \phi_{\omega} \mathbf{K}_{\omega}^{-1} \cdot \mathbf{V}_{\beta}^{*}+\mu_{\beta} \mathbf{K}_{\omega}^{-1} \cdot\left\{\widetilde{\mathbf{V}}_{\beta}^{\omega}\right\}_{\omega}
\end{aligned}
$$

Because the problem is fully linear, the above form suggests that we can express the Darcy-scale deviations as linear functions of the unique macroscopic source term $\mathbf{V}_{\beta}^{*}$ remaining in the closure problem. Thus, a macroscopically uniform flow over the representative unit cell can be described by the following mapping variables

$$
\begin{aligned}
& \tilde{\mathbf{V}}_{\beta}^{\omega}=\mathbf{B}_{\omega} \cdot \mathbf{V}_{\beta}^{*} \quad ; \quad \tilde{\mathbf{V}}_{\beta}^{\eta}=\mathbf{B}_{\eta} \cdot \mathbf{V}_{\beta}^{*} \\
& \tilde{P}_{\beta}^{\omega}=\mu_{\beta} \mathbf{b}_{\omega} \cdot \mathbf{V}_{\beta}^{*} \quad ; \quad \tilde{P}_{\beta}^{\eta}=\mu_{\beta} \mathbf{b}_{\eta} \cdot \mathbf{V}_{\beta}^{*}
\end{aligned}
$$

where $\mathbf{B}$ and $\mathbf{b}$ are, respectively, the closure tensor and closure vector of the system. Any additive constant in the relationships (56) and (57) would have no contribution to the final result as discussed in [64].

Introducing these representations into Eqs. 50-55, we obtain

\section{Closure problem}

$\nabla \cdot \mathbf{B}_{\omega}=0$

$0=-\nabla \mathbf{b}_{\omega}+\frac{1}{\varepsilon_{\omega}} \nabla^{2} \mathbf{B}_{\omega}-\mathbf{K}_{\omega}^{-1} \cdot\left(\phi_{\eta} \mathbf{I}+\mathbf{B}_{\omega}-\left\{\mathbf{B}_{\omega}\right\}_{\omega}\right)$

B.C. $1 \quad \mathbf{B}_{\omega}=\mathbf{B}_{\eta} \quad$ at $A_{\omega \eta}$

B.C. $2 \mathbf{n}_{\omega \eta} \cdot\left[\left(\mathbf{b}_{\eta}-\mathbf{b}_{\omega}\right) \mathbf{I}+\left(\frac{1}{\varepsilon_{\omega}} \nabla \mathbf{B}_{\omega}-\nabla \mathbf{B}_{\eta}\right)\right.$

$$
\left.+\left(\frac{1}{\varepsilon_{\omega}} \nabla \mathbf{B}_{\omega}-\nabla \mathbf{B}_{\eta}\right)^{T}\right]=0 \quad \text { at } A_{\omega \eta}
$$

$$
\nabla \cdot \mathbf{B}_{\eta}=0
$$

$0=-\nabla \mathbf{b}_{\eta}+\nabla^{2} \mathbf{B}_{\eta}+\mathbf{K}_{\omega}^{-1} \cdot\left(\phi_{\omega} \mathbf{I}+\left\{\mathbf{B}_{\omega}\right\}_{\omega}\right)$ 
$\mathbf{b}_{\kappa}(\mathbf{r}+\mathbf{l})=\mathbf{b}_{\kappa}(\mathbf{r}) ; \mathbf{B}_{\kappa}(\mathbf{r}+\mathbf{l})=\mathbf{B}_{\kappa}(\mathbf{r}) \kappa=\omega, \eta$

$\{\mathbf{b}\}=0$

The periodic boundary condition imposed by Eq. 64 is classically used in the volume averaging method. It must be seen more as a device for obtaining a local solution-because it usually imposes less severe constraints on the problem, than, for instance, a Dirichlet condition would - than a real limitation on the kind of medium under consideration. In other words, the use of periodic boundary conditions does not necessarily imply that the porous medium is periodic (see a discussion on this issue in [18]). Note also that Eq. 65 directly results from the average of Eq. 57, keeping in mind that the average of a spatial deviation is zero. Without this constraint, the problem would be solved to within an arbitrary but undetermined constant. Equation 56 leads to a similar condition of zero average for $\mathbf{B}$ but this one is not required for the explicit determination of the closure fields $\mathbf{b}$ and $\mathbf{B}$.

At this point, a more simple and numerically tractable version of the closure problem may be derived. This is achieved by transforming the closure variables $\mathbf{B}_{\kappa}, \mathbf{b}_{\kappa}$ into $\mathbf{D}_{\kappa}, \mathbf{d}_{\kappa},(\kappa=\omega, \eta)$ in the following manner

$\mathbf{b}_{\kappa}=\mathbf{d}_{\kappa} \cdot \mathbf{K}_{\omega}^{-1} \cdot\left(\phi_{\omega} \mathbf{I}+\left\{\mathbf{B}_{\omega}\right\}_{\omega}\right) \quad \kappa=\omega, \eta$

$\mathbf{B}_{\kappa}=\mathbf{D}_{\kappa} \cdot \mathbf{K}_{\omega}^{-1} \cdot\left(\phi_{\omega} \mathbf{I}+\left\{\mathbf{B}_{\omega}\right\}_{\omega}\right)-\mathbf{I} \quad \kappa=\omega, \eta$

In this way, the closure problem is given by

\section{Closure problem}

$\nabla \cdot \mathbf{D}_{\omega}=0$

$0=-\nabla \mathbf{d}_{\omega}+\frac{1}{\varepsilon_{\omega}} \nabla^{2} \mathbf{D}_{\omega}-\mathbf{K}_{\omega}^{-1} \cdot \mathbf{D}_{\omega}+\mathbf{I}$

B.C. $1 \quad \mathbf{D}_{\omega}=\mathbf{D}_{\eta} \quad$ at $A_{\omega \eta}$

B.C. $2 \quad \mathbf{n}_{\omega \eta} \cdot\left(\left(\mathbf{d}_{\eta}-\mathbf{d}_{\omega}\right) \mathbf{I}+\left(\frac{1}{\varepsilon_{\omega}} \nabla \mathbf{D}_{\omega}-\nabla \mathbf{D}_{\eta}\right)\right.$ $\left.+\left(\frac{1}{\varepsilon_{\omega}} \nabla \mathbf{D}_{\omega}-\nabla \mathbf{D}_{\eta}\right)^{T}\right)=0 \quad$ at $A_{\omega \eta}$

$$
\nabla \cdot \mathbf{D}_{\eta}=0
$$

$0=-\nabla \mathbf{d}_{\eta}+\nabla^{2} \mathbf{D}_{\eta}+\mathbf{I}$

$\mathbf{d}_{\kappa}(\mathbf{r}+\mathbf{l})=\mathbf{d}_{\kappa}(\mathbf{r}) ; \mathbf{D}_{\kappa}(\mathbf{r}+\mathbf{l})=\mathbf{D}_{\kappa}(\mathbf{r}) \kappa=\omega, \eta$

$$
\{\mathbf{d}\}=0
$$

Finally, it should be noted that the additional condition of zero average for $\mathbf{B}$ leads to the subsequent relation

$$
\{\mathbf{D}\}=\left(\phi_{\omega} \mathbf{I}+\left\{\mathbf{B}_{\omega}\right\}_{\omega}\right)^{-1} \cdot \mathbf{K}_{\omega}
$$

The closure problem expressed with $\mathbf{d}_{\kappa}$ and $\mathbf{D}_{\kappa},(\kappa=\omega$, $\eta)$ has clearly a Darcy-Brinkman-Stokes structure similar to the initial Darcy scale problem which means that a unique numerical tool can be used for both the direct solution of the flow problem in the $\omega$ and $\eta$ regions and the solution to the closure problem yielding the macroscopic coefficient that appears in the average momentum equation given in the following section. This closure problem is also consistent with the upscaling performed by Popov et al. [50] from a two-scale asymptotic expansion of the Darcy-Brinkman equation. To the best of our knowledge, this is the very first time that the closure relations governing the effective permeability of a vugular rock is rigorously derived within the framework of the volume averaging method.

\subsection{Large-scale momentum equation}

The macroscopic momentum equation is obtained while introducing the previous decomposition, Eq. 56, into the macroscopic Eq. 38. When the remapping Eq. 67 is used along with the relation (76), the following averaged equation is obtained

$0=-\nabla P_{\beta}^{*}-\mu_{\beta}\{\mathbf{D}\}^{-1} \cdot \mathbf{V}_{\beta}^{*}+\rho_{\beta} \mathbf{g}$

In this result, all the Brinkman terms in Eq. 38 have been neglected. The first one in the right-hand side of this equation can be discarded following an argument fully similar to that expressed in Eqs. 48 and 49. The two remaining ones, involving $\nabla \cdot\left\{\nabla \tilde{\mathbf{V}}_{\beta}^{\kappa}\right\}_{\kappa}(\kappa=\omega, \eta)$, can be completely neglected due to the scale contrast expressed in Eq. 43 along with the fact that the closure variables $\mathbf{B}_{\kappa}(\kappa=\omega, \eta)$ are periodic.

The averaged momentum equation, Eq. 77, can finally be rewritten as a large-scale Darcy's law given by

$\mathbf{V}_{\beta}^{*}=-\frac{\mathbf{K}_{e f f}^{*}}{\mu_{\beta}} \cdot\left(\nabla P_{\beta}^{*}-\rho_{\beta} \mathbf{g}\right)$

where the effective permeability $\mathbf{K}_{e f f}^{*}$ is given by

$\mathbf{K}_{\text {eff }}^{*}=\{\mathbf{D}\}$

Equation 78 is consistent with the form of the macroscopic model obtained by Arbogast and Lehr [1] from a two-scale homogenization of a Darcy-Stokes system, although their expression of the closure problem is different due to the use of the Beavers-Joseph boundary condition. A comparison between both solutions on a simple geometry will be performed in the next section.

\section{Results and discussion}

As discussed in the previous section, the solution to the closure problem is conducted over some representative periodic 
unit cells. As illustrative examples, two different geometries are considered here. Although simple, they are representative of natural systems (e.g., flow through a fracture or a vuggy porous medium) and provide useful information about the macroscopic flow behavior in such media.

The closure problem was solved using a commercial finite-element solver (COMSOL Multiphysics, Comsol, Inc., Los Angeles) on some model two-dimensional unit cells. Convergence analyses were conducted for these simulations to assure that the physics is appropriately resolved. Equations 68-75 were solved using a weak formulation to a prespecified relative convergence criterion. The permeability tensor was determined using the straightforward relationship of Eq. 79 .

\subsection{Case 1: stratified geometry}

The study of coupled parallel flows within a two-region domain is a classical problem in interfacial flow theory. Historically, the analysis of Poiseuille flow past over a porous block by Beavers and Joseph has led to the empirical derivation of the slip boundary condition as detailed in the introduction. Various configurations of stratified systems have been also subsequently studied within the framework of a Darcy-Brinkman equation (single-domain approach) or a double-domain approach with a Beavers-Joseph condition.

\subsubsection{Darcy-Brinkman approach}

Here, we consider the periodic stratified system illustrated in Fig. 3, where a fluid channel is embedded between two parallel porous layers. The interface between the two regions is parallel to the plane $y=0$ and the flow is one-dimensional in the $x$-direction. This kind of interfacial problem is interesting for several reasons.

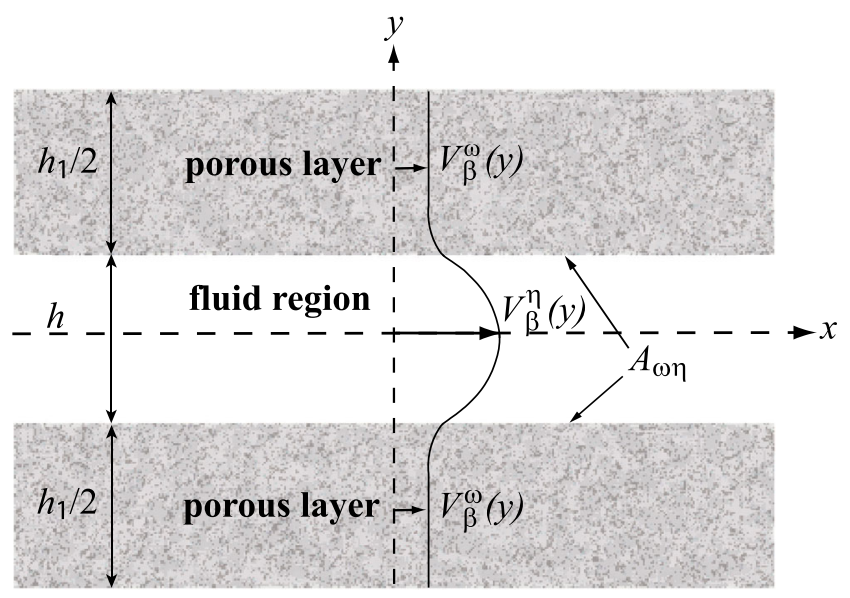

Fig. 3 Sketch of a porous-fluid domain with flow parallel to the layers
First, an explicit analytical solution is tractable that can be directly compared to the numerical solution of the closure problem given by Eqs. 68-75. For the configuration of Fig. 3 indeed, the boundary value problem describing the fluid-porous system takes the form:

$-\frac{d P}{d x}+\mu_{\beta} \frac{\partial^{2} V_{\beta}^{\eta}}{\partial y^{2}}=0$ in the $\eta$ region

$-\frac{d P}{d x}-\frac{\mu_{\beta}}{K_{\omega}} V_{\beta}^{\omega}+\frac{\mu_{\beta}}{\varepsilon_{\omega}} \frac{\partial^{2} V_{\beta}^{\omega}}{\partial y^{2}}=0$ in the $\omega$ region

B.C.1 $\quad V_{\beta}^{\eta}=V_{\beta}^{\omega}$ at $y= \pm \frac{h}{2}$

B.C.2 $\frac{\partial V_{\beta}^{\eta}}{\partial y}=\frac{1}{\varepsilon_{\omega}} \frac{\partial V_{\beta}^{\omega}}{\partial y}$ at $y= \pm \frac{h}{2}$

B.C.3 $V_{\beta}^{\eta}(y)=V_{\beta}^{\eta}\left(y+h+h_{1}\right)$

$$
\begin{gathered}
V_{\beta}^{\omega}(y)=V_{\beta}^{\omega}\left(y+h+h_{1}\right) \\
P(y)=P\left(y+h+h_{1}\right)
\end{gathered}
$$

Here, we have denoted $P$ the Darcy-scale pressure in both $\omega$ - and $\eta$-regions that is indeed independent of $y, \frac{d P}{d x}$ being the constant pressure applied to the system. The $x$ velocities $V_{\beta}^{\omega}$ and $V_{\beta}^{\eta}$ can be explicitly determined from the solution of this boundary value problem as shown in Appendix A. From these expressions, the effective permeability of the system, $K_{e f f}^{*}$, can be readily identified as:

$$
\begin{aligned}
K_{e f f}^{*}= & K_{\omega}+K_{\omega} \frac{h}{h+h_{1}}+\frac{h^{2}}{12}\left(\frac{h}{h+h_{1}}\right) \\
& +\sqrt{\varepsilon_{\omega} K_{\omega}} \frac{h^{2}}{2\left(h+h_{1}\right)} \frac{\left(1+\exp \left(-\sqrt{\frac{\varepsilon_{\omega}}{K_{\omega}}} h_{1}\right)\right)}{\left(1-\exp \left(-\sqrt{\frac{\varepsilon_{\omega}}{K_{\omega}}} h_{1}\right)\right)}
\end{aligned}
$$

As shown in Appendix A, the region-averaged permeabilities $K_{\omega}^{*}$ and $K_{\eta}^{*}$ can be also defined by:

$\bar{V}_{\beta}^{\eta}=\frac{1}{h} \int_{-h / 2}^{h / 2} V_{\beta}^{\eta} \mathrm{d} y=-\frac{K_{\eta}^{*}}{\mu_{\beta}} \frac{d P}{d x}$

$\bar{V}_{\beta}^{\omega}=\frac{1}{h_{1}} \int_{-\left(h / 2+h_{1}\right)}^{-h / 2} V_{\beta}^{\omega} \mathrm{d} y=-\frac{K_{\omega}^{*}}{\mu_{\beta}} \frac{d P}{d x}$

where $\bar{V}_{\beta}^{\eta}$ and $\bar{V}_{\beta}^{\omega}$ represent the two region-averaged intrinsic velocities. After derivation, we obtain:

$K_{\omega}^{*}=K_{\omega} \frac{\left(h_{1}+h\right)}{h_{1}}$

$K_{\eta}^{*}=\frac{h^{2}}{12}+K_{\omega}+\frac{\sqrt{\varepsilon_{\omega} K_{\omega}}}{2} h \frac{\left(1+\exp \left(-\sqrt{\frac{\varepsilon_{\omega}}{K_{\omega}}} h_{1}\right)\right)}{\left(1-\exp \left(-\sqrt{\frac{\varepsilon_{\omega}}{K_{\omega}}} h_{1}\right)\right)}$

Note that the effective permeability $K_{\omega}^{*}$ of the porous layer is larger than its intrinsic permeability $K_{\omega}$. This modification is due to the presence of the so-called Brinkman 
layer corresponding to an increase of the velocity at the vicinity of the fluid-porous interface. In other words, the porous zone may be seen as a two-region domain made of a porous layer of thickness $\left(h_{1}-h_{B}\right)$ and permeability $K_{\omega}$ and a more permeable (Brinkman) layer of thickness $h_{B}$ and effective permeability $K_{\omega} \frac{h+h_{B}}{h_{B}}$. As discussed in the introduction, theoretical studies [40,57] have shown that the thickness $h_{B}$ of this interfacial zone is of the order of $\sqrt{K_{\omega}}$, although the experimental work in [17] suggests that it might be much larger, of the order of the grain diameter. In the case under consideration, the influence of this transition zone appears not to be negligible and directly related to the ratio $h / h_{1}$. Indeed, for a fluid channel relatively thin compared to the thickness of the porous layer, i.e., for $h / h_{1} \rightarrow 0$, the intrinsic permeability of the porous medium, $K_{\omega}$, is recovered. We will discuss further this question of region averaged velocity in Section 4.1.3 after introducing the results obtained with the Beavers-Joseph boundary condition.

The first term of the fluid-averaged permeability, given by Eq. 89, corresponds to the classical permeability of a Poiseuille flow between two impermeable parallel plates. The two additional terms represent the correction due to the non-zero velocity boundary condition at $A_{\omega \eta}$. It should be emphasized that the exponential contribution which appears in the last term reflects the periodic boundary condition, Eq. 84, and characterizes the relative influence of the upper and lower fluid channels on the adjacent porous layer. For an infinite porous medium, i.e., $h_{1} \rightarrow \infty$, this correction disappears.

The comparison between this analytical solution and the computational results of $K_{\text {eff }}^{*}$ derived from the solution of the closure problem and the volume averaging technique is presented in Fig. 4 for a wide range of $K_{\omega}$ values. The following numerical data were used for the computations: $h=0.3, h_{1} x=0.7$, and $\varepsilon_{\beta}=0.3$. Note that the value of $K_{\eta}$ theoretically tending to infinity, was taken large enough in numerical simulations so that the resulting solution is unaltered. Typically, $K_{\eta}$ was taken equal to $10^{6}$. One observe a perfect agreement between the analytical and computational results. For small enough values of $K_{\omega}$ $\left(K_{\omega} \leq 10^{-6} \mathrm{~m}^{2}\right)$, the porous region can be considered as quasi-impermeable to longitudinal flow. The macroscopic permeability $K_{\text {eff }}^{*}$ remains constant and is mainly dependent upon the permeability of the channel, $h^{2} / 12$. When the matrix permeability increases, the contribution of the porous layers to the flow as well as the impact of the slip condition becomes significant. The solution obtained from a Darcy-Darcy approach is also represented on this figure for comparison. The equivalent permeability within the fluid region is taken equal to $h^{2} / 12$ while that in the $\omega$-region is taken uniformly equal to $K_{\omega}$. If solutions are similar for low values of $K_{\omega}$ (typically smaller than $10^{-5} \mathrm{~m}^{2}$ ), the

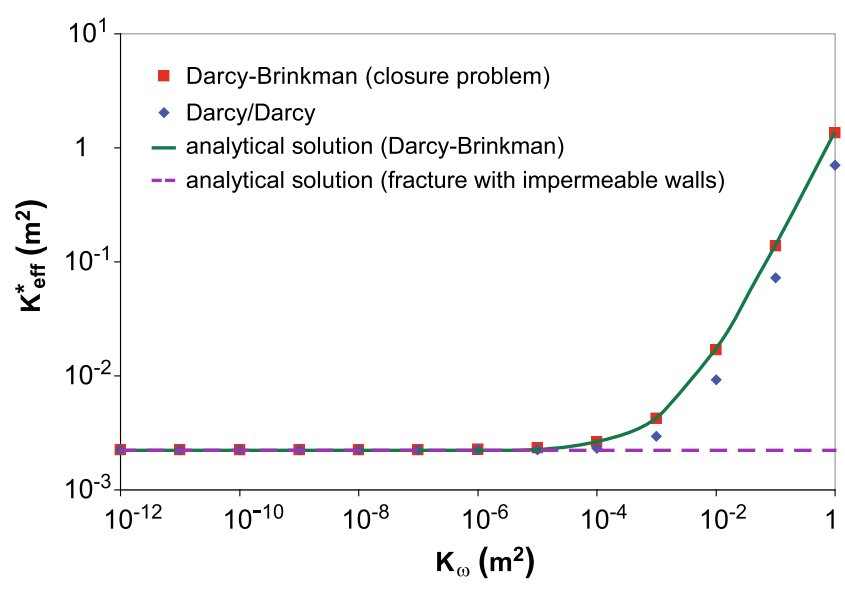

Fig. 4 Effective permeability, $K_{e f f}^{*}$, for a fractured medium or layered domain as a function of the porous region permeability, $\mathbf{K}_{\omega}$

contribution of both the slip-flow in the $\eta$-region at $A_{\omega \eta}$ and the Brinkman layer (not considered in the DarcyDarcy formulation) becomes significant for larger values of $K_{\omega}$ for which the Darcy-Darcy approach underestimates $K_{e f f}^{*}$.

\subsubsection{Comparison with the Darcy-Stokes approach (Beavers-Joseph formulation)}

If these results validate our upscaled model, they also confirm the difficulty of obtaining an appropriate and unique macroscopic representation of one-phase flow in such media. This uncertainty, due to the choice of the momentum transport model and to the associated coupling conditions at the interface, can be further enlighted through the comparison of the Darcy-Brinkman approach used here and the Beavers-Joseph formulation. Indeed, several authors [29, 40] have shown the similarity between both approaches for Poiseuille or Couette flow in layered systems in terms of the velocity profile within the fluid vein. As discussed in the introduction, for a Poiseuille-type flow (see Fig. 2i), this equivalence is verified provided one identifies the coefficient $\alpha$ in Eq. 8 with $\sqrt{\frac{\widetilde{\mu}}{\mu_{\beta}}}$. It shall be emphasized that this analogy, initially restricted to the geometry specified in Fig. 2 , is classically extrapolated to any fluid/porous configuration without any theoretical justification. Following this idea, a comparison of the solution for $K_{e f f}^{*}$, given by Eq. 85 , to the one obtained from the Darcy-Stokes approach (with a Beavers-Joseph boundary condition) can be carried out. Indeed, Arbogast and Lehr [1] recently derived a macroscopic model based on such an approach. A simple analytical solution is given to illustrate their results in the case of a periodic layered domain, similar to the one illustrated in Fig. 3. This solution, which can be directly 
compared to the one given by Eq. 85, is expressed as follows,

$$
\begin{aligned}
K_{e f f, B J}^{*}= & K_{\omega} \frac{h_{1}}{h+h_{1}}+\frac{h^{2}}{12}\left(\frac{h}{h+h_{1}}\right) \\
& +\frac{\sqrt{K_{\omega}}}{\alpha} \frac{h^{2}}{2\left(h+h_{1}\right)}
\end{aligned}
$$

Introducing the analogy condition cited above for a Poiseuille flow,

$$
\alpha=\sqrt{\frac{1}{\varepsilon_{\omega}}}
$$

we finally obtain

$$
\begin{aligned}
K_{e f f, B J}^{*}= & K_{\omega} \frac{h_{1}}{h+h_{1}}+\frac{h^{2}}{12}\left(\frac{h}{h+h_{1}}\right) \\
& +\sqrt{\varepsilon_{\omega} K_{\omega}} \frac{h^{2}}{2\left(h+h_{1}\right)}
\end{aligned}
$$

This result can also be interpretated in terms of regionaveraged permeabilities which leads to the following expressions for $K_{\eta, B J}^{*}$ and $K_{\omega, B J}^{*}$ :

$K_{\omega, B J}^{*}=K_{\omega}$

$K_{\eta, B J}^{*}=\frac{h^{2}}{12}+\frac{\sqrt{\varepsilon_{\omega} K_{\omega}}}{2} h$

The comparison of these expressions with Eqs. 88 and 89 clearly shows that the analogy condition is violated. In the porous region, as expected, the intrinsic permeability (i.e., without the additional term associated to the Brinkman layer) is recovered, whereas the fluid region permeability exhibits a correction term that is different, although small if $K_{\omega}$ is small. Apparently, in the case under consideration, the hydrodynamic coupling between the different layers is more complex than for a classical Poiseuille flow with an immobile wall and induces a discrepancy between the solutions. This clearly indicates that the condition on $\alpha$ (see Eq. 8) for the analogy mentioned above should be used with caution even for layered media. Nevertheless, this should be relativized since this difference has a significant impact on the effective permeability only for large values of both $K_{\omega}$ and the relative fluid channel thickness, as illustrated in Fig. 5. Three distinct values of the $\eta$-region volume fraction, $\phi_{\eta}$, (i.e., the ratio of $h$ to $h+h_{1}$ ) were considered while $\varepsilon_{\omega}$ fixed to 0.2 .

\subsubsection{Discussion on the porous medium regional velocity predicted by the Darcy-Brinkman formulation}

Regional velocities play an important role in porous media modeling when dual-media effects are important. This is classically the case while working with dual-heterogeneous media, i.e., when upscaling from a Darcy scale to a large

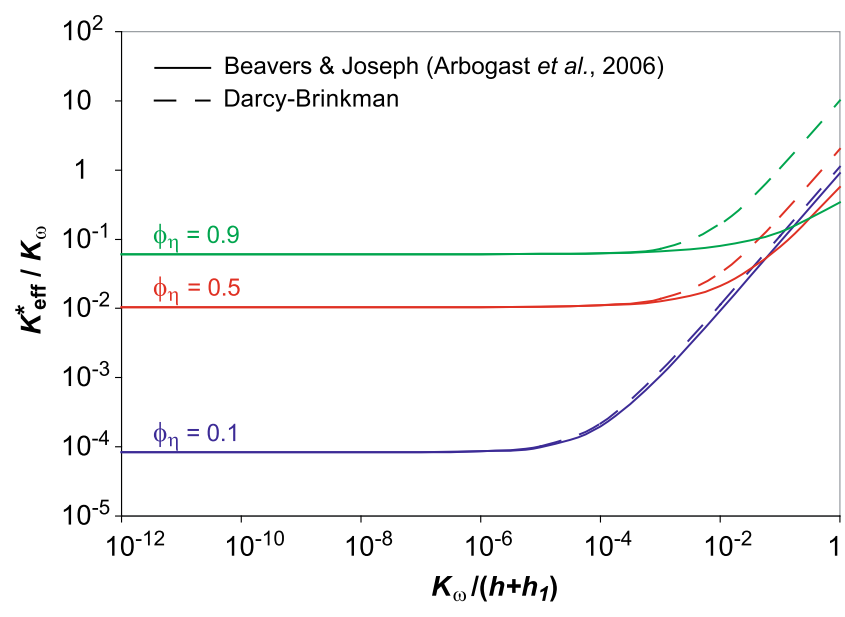

Fig. 5 Comparison of effective permeabilities obtained from the Darcy-Brinkman and Beavers-Joseph formulations for the layered system of Fig. 3. Values of the parameters are those for Fig. 4

scale. Indeed, regional velocities play a key role for solute transport in generalized dual-porosity models, the so-called mobile-mobile systems, as discussed in $[10,11,16]$. The theoretical derivation of regional Darcy's laws and regional permeabilities is discussed in [51]. Regional Darcy's laws may also arise from pore-scale to Darcy-scale upscaling problem as discussed in [55]. In the case of a stratified heterogeneous porous medium, the $\omega$-region permeability was given by $K_{\omega}^{*}=\phi_{\omega} K_{\omega}$ in [51] with a different definition of the regional velocity, namely in terms of a large-scale filtration velocity. If we adopt the definition used in this paper, this result can be recast into $K_{\omega}^{*}=K_{\omega}$ which is exactly equal to the result for $K_{\omega, B J}^{*}$ in Eq. 93. Why is the Darcy-Brinkman result so different? As indicated above, Darcy-Brinkman equations generate a kind of viscous boundary layer of thickness $h_{B}$ that penetrates into the porous domain. This is illustrated in Fig. 6 which represents the velocity variation in the lower $\omega$-region versus the reduced position $y_{r}=(y+h / 2) / h_{1}$ for different values of the permeability. Here, the velocity $V_{\beta}^{\omega}$ (Eq. 100) is reduced by the reference velocity $V_{D}^{\omega}$ classically obtained from Darcy's law:

$V_{D}^{\omega}=-\frac{K_{\omega}}{\mu_{\beta}} \frac{d P}{d x}$

Two mechanisms may be observed. First, the thickness of the Brinkman boundary layer, i.e., the zone where $V_{\beta}^{\omega} \neq$ $V_{D}^{\omega}$, increases with the permeability. To be more precise about this variation, we arbitrarily define the extent of this viscous layer relative to the velocity $V_{\beta}^{\omega}$ within the zone such that

$V_{\beta}^{\omega} \geq 1.01 V_{D}^{\omega}$

and we identify the value of $y_{r}$ at which this condition is fulfilled as $h_{B} / h_{1}$. The behavior of $h_{B} / h_{1}$ as a function 


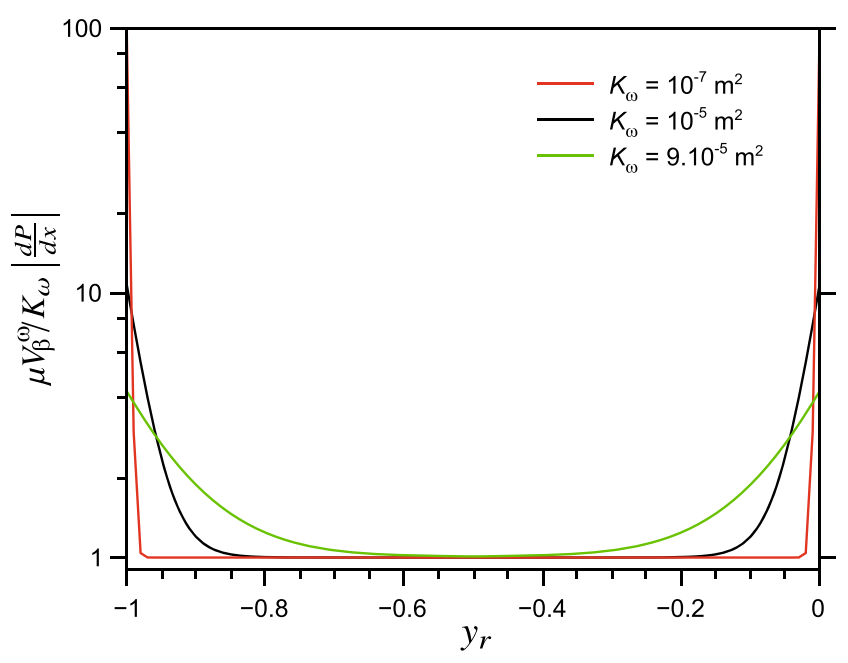

Fig. 6 Dimensionless velocity as a function of the reduced position for various permeabilities $\left(\varepsilon_{\omega}=0.38\right)$

of $\sqrt{K_{\omega}}$ is represented in Fig. 7. The viscous layer starts from 0 at exceedingly small values of $K_{\omega}$ and increases, after a transient stage at low permeability, linearly with $\sqrt{K_{\omega}}$ and, eventually, changes its behavior to reach the middle of the porous stratum for a given value of $K_{\omega}$. At this point, the boundary layer occupies the entire porous domain and Darcy's law is nowhere verified. The second important aspect is that the measure of the viscous layer goes to zero as the permeability decreases; however, the velocity inside goes up such that the average velocity (or flow-rate) remains finite within the $\omega$-region. This in turn gives a finite constant contribution to the regional velocity and the regional permeability, as shown in Eq. 88. This may be a problem when using the Darcy-Brinkman solution to investigate the flow of a solute in the porous region since the advection

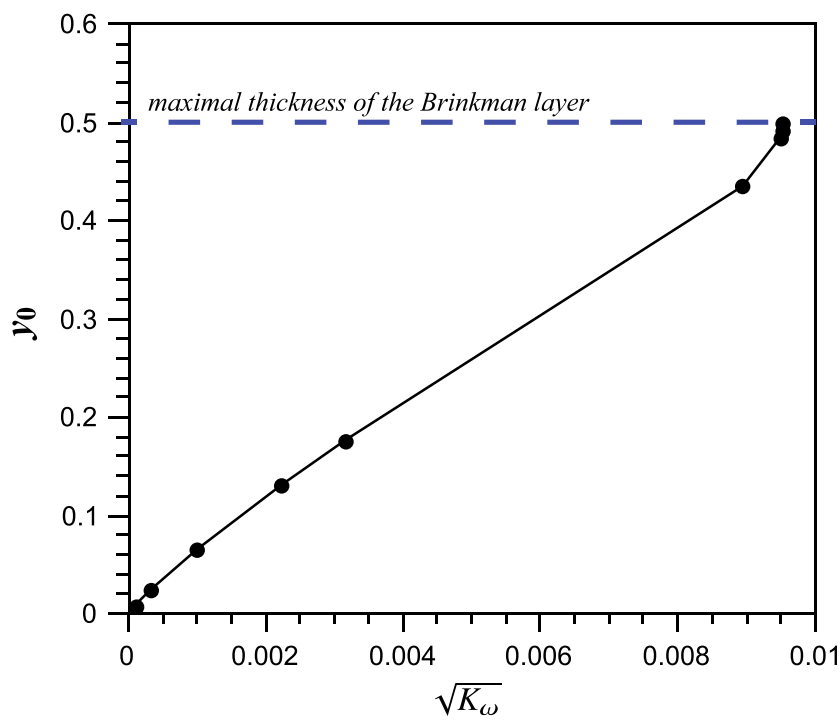

Fig. 7 Viscous layer thickness as a function of $\sqrt{K_{\omega}}\left(\varepsilon_{\omega}=0.38\right)$ velocity affected to the $\omega$-region equation would be largely overestimated. On the contrary, the analysis with the Beavers-Joseph boundary condition does not suffer from this effect.

\subsection{Case 2: 2D vuggy medium}

The configuration under consideration in this section consists of a two-dimensional periodic vuggy system made of circular fluid inclusion arranged on a regular square array embedded in a porous matrix. The representative unit cell of length $\ell$ is depicted in Fig. 8. The system is far more complex to be solved analytically, and some relatively accurate approximated solutions were proposed (and checked experimentally) in [32]. Additional analytical or semi-analytical solutions based on single-domain or double-domain approaches and simplifying assumptions can also be drawn from the literature. Recently, the velocity field was explicitly determined in this configuration for a Darcy-Brinkman flow of a viscous fluid through a circular-porous or fluid-filled-inclusion embedded in another porous medium [22]. In [36], a Darcy-Stokes model coupled with a Saffman boundary condition was employed to calculate the permeability of an equivalent Darcy-type inclusion of spherical and circular shapes. The effective global permeability of the associated vugular system was then determined by using Maxwell's method. In the case of circular inclusions, the following relation is obtained:

$$
\begin{array}{r}
K_{\text {eff }, \text { vug }}^{*}=\frac{K_{\omega}\left[R^{2}\left(1+\phi_{\eta}\right)-2\left(\phi_{\eta}-1\right) K_{\omega}\right.}{R^{2}\left(1-\phi_{\eta}\right)+2\left(\phi_{\eta}+1\right) K_{\omega}} \\
\cdots \frac{\left.-3 R\left(1+\phi_{\eta}\right) \alpha \sqrt{K_{\omega}}\right]}{+3 R\left(\phi_{\eta}-1\right) \alpha \sqrt{K_{\omega}}}
\end{array}
$$

where $R$ represents the vug radius. Given the periodic nature of the system under consideration, vug radius is directly related to the value of the fluid volume fraction as

$$
R=\sqrt{\frac{\phi_{\eta}}{\pi}} \ell
$$

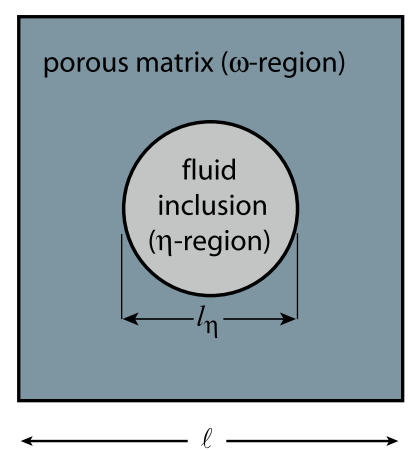

Fig. 8 Representative unit cell of a model 2D vuggy porous medium 


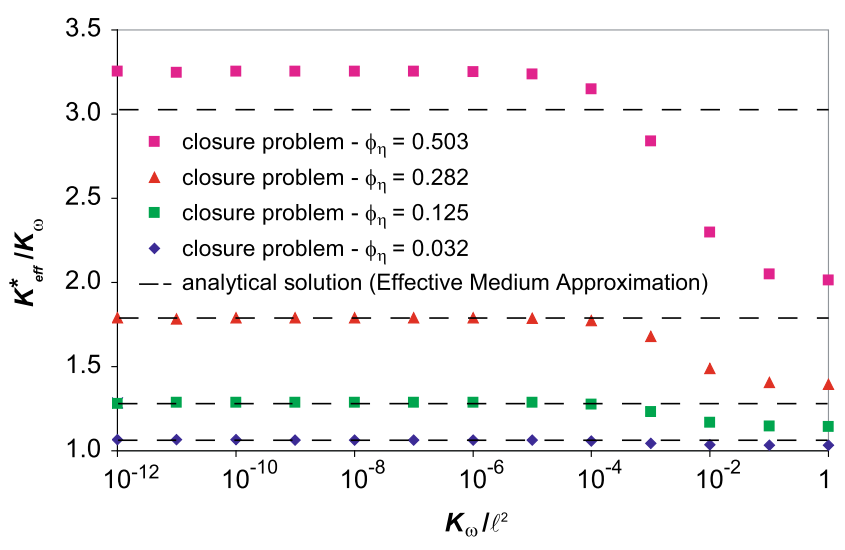

Fig. 9 Dimensionless effective permeability for a porous medium with circular fluid inclusions versus the dimensionless porous region permeability

Comparison between our computational results and this latter solution is illustrated in Fig. 9 where we have represented the variation of $K_{e f f}^{*} / K_{\omega}$ as a function of the dimensionless matrix permeability $K_{\omega} / \ell^{2}$ for different values of $\phi_{\eta}$. Here, $\alpha$ is arbitrarily fixed to $\sqrt{1 / \varepsilon_{\beta}}$ with $\varepsilon_{\omega}=$ 0.3 even if the analogy condition of [40] is probably not verified for this kind of geometry.

The constant solution resulting from Eq. 97 for a given value of $\phi_{\eta}$ deserves a few additional comments. Even though this relation seems to depend in a complex manner on $K_{\omega}$, the behavior of $K_{e f f, v u g}^{*}$ is quasi-linear (i.e., the ratio $K_{e f f, v u g}^{*} / K_{\omega}$ is quasi-constant) except for large values of $K_{\omega}$ for which the relation is not verified anymore. Indeed, application of Saffman's boundary condition to curved interfaces requires $R \gg \sqrt{K_{\omega}}$. Consequently, on this figure, we have represented the asymptotic behavior of $K_{e f f, v u g}^{*}$ only. For $K_{\omega} / \ell^{2} \lesssim 10^{-4}$, good agreement between both results is achieved in spite of the difference of treatment of interfacial conditions. This similarity is verified especially at low volume fractions of the fluid region. For large values of $\phi_{\eta}$, indeed, the assumption $R \ll \ell$ is not fulfilled (cf. Eq. 98) and hence, the Effective medium approximation fails.

\section{Conclusion}

In this paper, the interest is focused on the macroscopic behavior of one-phase flow in fractured and vuggy porous media. Volume averaging method is used to derive a largescale flow model from a Darcy-Brinkman description at the Darcy scale. Provided some length-scale constraints are satisfied, it is shown that the incompressible flow through a fractured or vuggy rock can be described by a macroscopic Darcy equation. This result is consistent with formulation obtained in [1] from homogenization of a Darcy-Stokes system with a Beavers-Joseph interfacial condition and in [50]. The effective permeability can be determined from the medium structure by solving the closure problem that is derived in this paper. Calculations of this large-scale permeability for different geometries and comparisons with theoretical solutions show that:

- The equivalence between the present Darcy-Brinkman approach and the two-domain (Beavers-Joseph) approach that is theoretically justified on the Poiseuilletype configuration —one wall of the fluid channel being impervious-and which makes use of the analogy between $\alpha$ in the Beavers-Joseph boundary condition and $\tilde{\mu}$ in the Brinkman term, must be employed with caution, although it has been used empirically in an extensive manner in many other configurations. In particular, region-averaged velocities and associated regional permeabilities predicted by both methods may be significantly different under certain conditions.

- Despite this lack of equivalence, effective permeabilities obtained from the two approaches are extremely close in practice, except at exceedingly large values of the permeability of the porous region.

More insight could be gained from the analysis of both flux and stress jump conditions at the porous-fluid interface in a Darcy-Brinkman-Stokes approach.

\section{Nomenclature}

\section{Roman Letters}

$\mathcal{A}_{\omega \eta} \quad$ Area of the interface $A_{\omega \eta}$ between the $\omega$-region and the $\eta$ - region, $\mathrm{m}^{2}$

$\mathbf{d}_{\kappa} \quad$ Closure variable mapping $\mathbf{V}_{\beta}^{*}$ onto $\tilde{P}_{\beta}^{\kappa}(\kappa=\omega, \eta)$ as defined in Eq. 66, $\mathrm{m} / \mathrm{s}$

$\mathbf{D}_{\kappa} \quad$ Closure variable mapping $\mathbf{V}_{\beta}^{*}$ onto $\widetilde{\mathbf{V}}_{\beta}^{\kappa}(\kappa=\omega, \eta)$ as defined in Eq. $67, \mathrm{~m}^{2}$

$\mathbf{K}_{\omega} \quad$ Permeability tensor in the porous matrix, $\mathrm{m}^{2}$

$\mathbf{K}_{e f f}^{*} \quad$ Effective permeability tensor for the vuggy porous medium, $\mathrm{m}^{2}$

L Characteristic length associated with the macroscale, as defined on Fig. 1, m

$\ell_{\beta} \quad$ Characteristic length associated with the pore-scale, as defined on Fig. 1, m

$\ell_{\sigma} \quad$ Characteristic length associated with the pore-scale, as defined on Fig. 1, $\mathrm{m}$

$l_{\kappa} \quad$ Characteristic length in the $\kappa$-region $(\kappa=\omega, \eta)$ associated with the Darcy-scale (Fig. 1), m

$\mathbf{n}_{\omega \eta} \quad$ Unit normal vector at $A_{\omega \eta}$, pointing from the $\omega$-region toward the $\eta$-region

$p_{\beta} \quad$ Fluid pressure in the $\beta$-phase, $\mathrm{N} / \mathrm{m}^{2}$

$P_{\beta}^{\kappa} \quad$ Intrinsic averaged pressure in the $\kappa$-region $(\kappa=\omega, \eta), \mathrm{N} / \mathrm{m}^{2}$ 
$\widetilde{P}_{\widetilde{P}^{\beta}}^{*} \quad$ Averaged fluid pressure in the vuggy rock, $\mathrm{N} / \mathrm{m}^{2}$

$\widetilde{P}_{\beta}^{\beta} \quad$ Large-scale pressure deviation in the vuggy or fractured medium, $\mathrm{N} / \mathrm{m}^{2}$

$\mathbf{v}_{\beta} \quad$ Fluid velocity in the $\beta$-phase, $\mathrm{m} / \mathrm{s}$

$\mathbf{V}_{\beta}^{\kappa} \quad$ Filtration velocity in the the $\kappa$-region $(\kappa=\omega, \eta)$, $\mathrm{m} / \mathrm{s}$

$\mathbf{V}_{\beta}^{*} \quad$ Large-scale superficial averaged fluid velocity in the vuggy or fractured medium, $\mathrm{m} / \mathrm{s}$

$V \quad$ Large-scale averaging volume, $\mathrm{m}^{3}$

$V_{\kappa} \quad$ Fraction of the large-scale averaging volume contained in the $\kappa$-region $(\kappa=\omega, \eta), \mathrm{m}^{3}$

\section{Greek letters}

$\alpha \quad$ Jump coefficient as defined by Eq. 8

$\varepsilon_{\omega} \quad$ Porosity in the $\omega$-region

$\mu_{\beta} \quad$ Fluid dynamic viscosity, $P$ a $s$

$\rho_{\beta} \quad$ Density, $\mathrm{kg} / \mathrm{m}^{3}$

$\phi_{\kappa} \quad$ Volume fraction of the $\kappa$-region, $(\kappa=\omega, \eta)$

\section{Subscripts}

$\beta \quad$ Fluid phase

$\omega$ Region associated to the porous medium

$\eta \quad$ Region associated to the fluid inclusions (vugs) or fluid-filled fractures

\section{Appendix A: Analytical solution of the Darcy-Brinkman equation in a stratified medium}

In this appendix, we detail the solution of the DarcyBrinkman flow within a stratified medium depicted in Fig. 3. We consider a steady incompressible flow in a twodimensional domain made of a fluid channel of thickness $h$ between two porous layers of height $h_{1} / 2$ each, as illustrated in Fig. 3. The medium is periodic along the $y$ direction and a constant pressure gradient is imposed along the $x$ direction. Flow in both zones is assumed to be governed by the boundary value problem given by Eqs. 80 to 84 . Given the problem geometry and boundary conditions, the flow is one-dimensional and velocity fields depend only on the $y$ coordinate.

Longitudinal velocities $V_{\beta}^{\eta}$ and $V_{\beta}^{\omega}$ can be easily determined for such a geometry. The solution within the fluid region, i.e., $-h / 2 \leq y \leq h / 2$, leads to:

$$
\begin{aligned}
V_{\beta}^{\eta}= & \frac{y^{2}}{2}-h \sqrt{\varepsilon_{\omega} K_{\omega}} \frac{1+\exp \left(-\sqrt{\frac{\varepsilon_{\omega}}{K_{\omega}}} h_{1}\right)}{2\left(1-\exp \left(-\sqrt{\frac{\varepsilon_{\omega}}{K_{\omega}}} h_{1}\right)\right)} \\
& \left.-\frac{h^{2}}{8}-K_{\omega}\right] \frac{1}{\mu_{\beta}} \frac{d P}{d x} \quad-h / 2 \leq y \leq h / 2
\end{aligned}
$$

The solution in the porous $\omega$-region is obtained similarly and takes the following form for $y \leq-h / 2$ :

$$
V_{\beta}^{\omega}=-\left[K_{\omega}+\frac{h}{2} \beta_{\omega} \sqrt{\varepsilon_{\omega} K_{\omega}}\right] \frac{1}{\mu_{\beta}} \frac{d P}{d x} \quad y \leq-h / 2
$$

where $\beta_{\omega}$ is defined by

$\beta_{\omega}=\frac{\exp \left(\sqrt{\frac{\varepsilon_{\omega}}{K_{\omega}}} y\right)+\exp \left(-\sqrt{\frac{\varepsilon_{\omega}}{K_{\omega}}}\left(h+h_{1}+y\right)\right)}{\exp \left(-\sqrt{\frac{\varepsilon_{\omega}}{K_{\omega}}} \frac{h}{2}\right)\left(1-\exp \left(-\sqrt{\frac{\varepsilon_{\omega}}{K_{\omega}}} h_{1}\right)\right)}$

From these two results, the region-averaged velocities $\bar{V}_{\beta}^{\eta}$ and $\bar{V}_{\beta}^{\omega}$ can be calculated as follows:

$$
\bar{V}_{\beta}^{\eta}=\frac{1}{h} \int_{-h / 2}^{h / 2} V_{\beta}^{\eta} \mathrm{d} y \quad ; \quad \bar{V}_{\beta}^{\omega}=\frac{1}{h_{1}} \int_{-\left(h / 2+h_{1}\right)}^{-h / 2} V_{\beta}^{\omega} \mathrm{d} y
$$

and, after integration, one can identify the region-averaged permeabilities, $K_{\eta}^{*}$ and $K_{\omega}^{*}$, by analogy with Darcy's law, such as

$K_{\eta}^{*}=\frac{h^{2}}{12}+K_{\omega}+\frac{\sqrt{\varepsilon_{\omega} K_{\omega}}}{2} h \frac{\left(1+\exp \left(-\sqrt{\frac{\varepsilon_{\omega}}{K_{\omega}}} h_{1}\right)\right)}{\left(1-\exp \left(-\sqrt{\frac{\varepsilon_{\omega}}{K_{\omega}}} h_{1}\right)\right)}$

$K_{\omega}^{*}=K_{\omega} \frac{\left(h_{1}+h\right)}{h_{1}}$

At this point, the global effective permeability $K_{\text {eff }}^{*}$ for the domain under consideration can be directly derived by a simple weighted arithmetic average for a stratified system to give

$K_{e f f}^{*}=\frac{h_{1} K_{\omega}^{*}+h K_{\eta}^{*}}{\left(h+h_{1}\right)}$

Substituting the solution of $K_{\eta}^{*}$ and $K_{\omega}^{*}$, given by Eqs. 103 and 104, respectively, into Eq. 105 and rearranging the terms, we finally obtain

Effective permeability associated to the layered domain

$$
\begin{aligned}
K_{e f f}^{*}= & K_{\omega}+K_{\omega} \frac{h}{h+h_{1}}+\frac{h^{2}}{12}\left(\frac{h}{h+h_{1}}\right) \\
& +\sqrt{\varepsilon_{\omega} K_{\omega}} \frac{h^{2}}{2\left(h+h_{1}\right)} \frac{\left(1+\exp \left(-\sqrt{\frac{\varepsilon_{\omega}}{K_{\omega}}} h_{1}\right)\right)}{\left(1-\exp \left(-\sqrt{\frac{\varepsilon_{\omega}}{K_{\omega}}} h_{1}\right)\right)}
\end{aligned}
$$

\section{References}

1. Arbogast, T., Lehr, H.: Homogenization of a Darcy-Stokes system modeling vuggy pous media. Comput. Geosci. 10(3), 291302 (2006)

2. Arquis, E., Caltagirone, J.: Sur les conditions hydrodynamiques au voisinage d'une interface milieu fluide-milieu poreux : Application à la convection naturelle. C.R. Acad. Sciences Série IIb 299, 1-4 (1984)

3. Bai, H., Yu, P., Winoto, S., Low, H.: Boundary conditions at the interface between fluid layer and fibrous medium. Int. J. Numer. Meth. Fluids 60, 809-825 (2009)

4. Beavers, G., Joseph, D.: Boundary conditions at a naturally permeable wall. J. Fluid Mech 30(1), 197-207 (1967) 
5. Beckermann, C., Viskanta, R., Ramadhyani, S.: A numerical study of non-darcian natural convection in a vertical enclosure filled with a porous medium. Numerical Heat Transfer 10(6), 557-570 (1986)

6. Beckermann, C., Viskanta, R., Ramadhyani, S.: Natural convection in vertical enclosures containing simultaneously fluid and porous layers. J. Fluid Mech. 186, 257-284 (1988)

7. Brinkman, H.: A calculation of the viscous force exerted by a flowing fluid on a dense swarm of particles. Appl. Sci. Res. 1, 2734 (1947)

8. Casar-Gonzalez, R.: Stochastic imaging of vuggy formations. In: SPE International Petroleum Conference and Exhibition, Mexico, 1-3 February 2000, SPE 58998 (2000)

9. Chandesris, M., Jamet, D.: Jump conditions and surface-excess quantities at a fluid/porous interface: a multi-scale approach. Transp. Porous Media 78, 419-438 (2009)

10. Cherblanc, F., Ahmadi, A., Quintard, M.: Two-medium description of dispersion in heterogeneous porous media: calculation of macroscopic properties. Water Resources Res. 39(6), 1154 (2003). doi:10.1029/2002WR001559

11. Cherblanc, F., Ahmadi, A., Quintard, M.: Two-domain description of solute transport in heterogeneous porous media: comparison between theoretical predictions and numerical experiments. Adv. Water Resour. 30(5), 1127-1143 (2007)

12. Dauba, C., Hamon, G., Quintard, M., Lasseux, D.: Stochastic description of experimental $3 \mathrm{~d}$ permeability fields in vuggy reservoir cores. Symp. Soc. Core Analysts, 13-16 September, The Hague, The Netherlands (1998)

13. de Zabala, E., Kamath, J.: Laboratory evaluation of waterflood behavior of vugular carbonates. In: SPE Annual Technical Conference and Exhibition, Dallas, 22-25 October 1995, SPE 30780 (1995)

14. Dehghani, K., Edwards, K., Harris, P.: Modeling of waterflood in a vuggy carbonate reservoir. In: SPE Annual Technical Conference and Exhibition, San Antonio, 5-8 October 1997, SPE 38910 (1997)

15. Ene, H., Sanchez-Palencia, E.: Equations et phénomènes de surface pour l'écoulement dans un modèle de milieu poreux. J. Méca 14, 73-108 (1975)

16. Gerke, H.H., VanGenuchten, M.T.: A dual-porosity model for simulating the preferential movement of water and solutes in structured porous media. Water Resour. Res. 29, 305-319 (1993)

17. Goharzadeh, A., Khalili, A., Jorgensen, B.: Transition layer thickness at a fluid-porous interface. Phys. Fluids 17(5), 057,102.1057,102.10 (2005)

18. Golfier, F., Wood, B., Orgogozo, L., Quintard, M., Buès, M.: Biofilms in porous media: development of macroscopic transport equations via volume averaging with closure for local mass equilibrium conditions. Adv. Water Resour. 32, 463-485 (2009)

19. Golfier, F., Zarcone, C., Bazin, B., Lenormand, R., Lasseux, D., Quintard, M.: On the ability of a Darcy-scale model to capture wormhole formation during the dissolution of a porous medium. J. Fluid Mech. 457, 213-254 (2002)

20. Goyeau, B., Lhuillier, D., Gobin, D., Velarde, M.: Momentum transport at a fluid-porous interface. Int. J. Heat Mass Transfer 46, 4071-4081 (2003)

21. Gray, W.: A derivation of the equations for multi-phase transport. Chem. Engng. Sci. 30, 229-233 (1975)

22. Grosan, T., Postelnicu, A., Pop, I.: Brinkman flow of a viscous fluid through a spherical porous medium embedded in another porous medium. Transp. Porous Media 81(1), 89-103 (2010)

23. Howes, F., Whitaker, S.: The spatial averaging theorem revisited. Chem. Engng Sci. 40, 1387-1392 (1985)

24. Huang, Z., Yao, J., Li, Y., Wang, C., Lv, X.: Numerical calculation of equivalent permeability tensor for fractured vuggy porous media based on homogenization theory. Commun. Comput. Phys. 9(1), 180-204 (2011)

25. Jäger, W., Mikelić, A.: On the interface boundary condition of Beavers, Joseph and Saffman. SIAM J. Appl. Math. 60, 11111127 (1990)

26. Jamet, D., Chandesris, M., Goyeau, B.: On the equivalence of the discontinuous one- and two-domain approaches for the modeling of transport phenomena at a fluid/porous interface. Transp. Porous Media 78, 403-418 (2009)

27. Jones, I.: Low Reynolds number flow past a porous spherical shell. Proc. Camb. Phil. Soc. 73, 231-238 (1973)

28. Joseph, D., Tao, L.: Lubrication of a porous bearing-Stokes' solution. J. App. Mech. 33(4), 753-760 (1966)

29. Koplik, J., Levine, H., Zee, A.: Viscosity renormalization in the Brinkman equation. Phys. Fluids 26(10), 2864-2870 (1983)

30. Laptev, V.: Numerical Solution of Coupled Flow in Plain and Porous Media. Ph.D. thesis, University of Kaiserslautern (2003)

31. Lasseux, D., Dauba, C., Quintard, M., Hamon, G., Prinet, C.: Local permeability measurement in vuggy rocks using minipermeameter: a numerical investigation. Gordon Research Conference, August, Andover, USA (1998)

32. Lasseux, D., Pairoys, F., Bertin, H.: Fluid flow in vugular porous media. In: Poro-Mechanics II, pp. 481-487. Balkema, Lisse (2002)

33. Lucia, F.: Carbonate reservoir characterization. Springer, New York (1999)

34. Lundgren, T.: Slow flow through stationnary random beds and suspensions of spheres. J. Fluid Mech. 51(2), 273-299 (1972)

35. Marciniak-Czochra, A., Mikelic, A.: Effective pressure interface law for transport phenomena between an unconfined fluid and a porous medium using homogenization. SIAM: Multiscale Model. Simul. 10(2), 285-305 (2012)

36. Markov, M., Kazatchenko, E., Mousatov, A., Pervago, E.: Permeability of fluid-filled inclusions in porous media. Trans. Porous Media 84(2), 307-317 (2010)

37. Martys, N., Bentz, D., Garboczi, E.: Computer simulation study of the effective viscosity in Brinkman's equation. Phys. Fluids 6(4), 1434-1439 (1994)

38. Moctezuma-Berthier, A., Fleury, M.: Permeability mapping on vuggy core sample using tracer experiments and streamline simulations. In: SPE International Petroleum Conference and Exhibition, Mexico, 1-3 February 2000, SPE 58992 (2000)

39. Moctezuma-Berthier, A., Vizika, O., Thovert, J., Adler, P.: Oneand two-phase permeabilities of vugular porous media. Trans. Porous Media 56, 225-244 (2004)

40. Neale, G., Nader, W.: Practical significance of Brinkman's extension of Darcy's law: Coupled parallel flows within a channel and a bounding porous medium. Canad. J. Chem. Eng. 52, 475-478 (1974)

41. Nield, D.: The boundary correction for the Rayleigh-Darcy problem: Limitation of the Brinkman equation. J. Fluid Mech. 128, 37 46 (1983)

42. Nield, D.: The Beavers-Joseph boundary condition and related matters: A historical and critical note. Trans. Porous Media 78, 537-540 (2009)

43. Nilson, R., Griffiths, S.: Wormhole growth in soluble porous materials. Phys. Rev. Lett. 65(13), 1583-1586 (1990)

44. Ochoa-Tapia, J., Whitaker, S.: Momentum transfer at the boundary between a porous medium and a homogeneous fluid-I. Theoretical development. I.t. J. Heat Mass Transfer 38, 2635-2646 (1995)

45. Ochoa-Tapia, J., Whitaker, S.: Momentum transfer at the boundary between a porous medium and a homogeneous fluid-II. Comparison with experiment. Int. J. Heat Mass Transfer 38, 2647-2655 (1995) 
46. Okabe, H., Blunt, M.J.: Pore space reconstruction of vuggy carbonates using microtomography and multiple-point statistics. Water Resour. Res. 43, W12S02 (2007). doi:10.1029/ 2006WR005,680

47. Padhy, G., Ioannidis, M., Lemaire, C.: Special core analysis studies in vuggy porous media of controlled microstructure. In: International Symposium of the Society of Core Analysts, Toronto, 21-25 August 2005 (2005)

48. Pairoys, F., Lasseux, D., Bertin, H.: An experimental and numerical investigation of water-oil flow in vugular porous media. Symp. Soc. Core Analysts, 21-24 September, Pau, France (2003)

49. Panga, M., Ziauddin, M., Balakotaiah, V.: Two-scale continuum model for simulation of wormholes in carbonate acidization. AIChE J 51(12), 3231-3248 (2005)

50. Popov, P., Efendiev, Y., Qin, G.: Multiscale modeling and simulations of flows in naturally fractured karst reservoirs. Commun. Comput. Phys. 6, 162-184 (2009)

51. Quintard, M., Whitaker, S.: Transport in chemically and mechanically heterogeneous porous media III: large-scale mechanical equilibrium and the regional form of Darcy's law. Adv. Water Resour. 21, 617-629 (1998)

52. Reza Morad, M., Khalili, A.: Transition layer thickness in a fluidporous medium of multi-sized sperical beads. Exp. Fluids 46, 323 330 (2009)

53. Saffman, P.: On the boundary condition at the surface of a porous medium. Stud. Appl. Math. 50, 93-101 (1971)

54. Sahraoui, M., Kaviany, M.: Slip and no-slip velocity boundary conditions at interface of porous, plain media. Int. J. Heat Mass Transp 35, 927-943 (1992)
55. Soulaine, C., Davit, Y., Quintard, M.: A two-pressure model for slightly compressible single phase flow in bistructured porous media. Chem. Eng. Sci. 96, 55-70 (2013). doi: 10.1016/j.ces.2013.03.060

56. Spaid, M., Phelan Jr. F.: Lattice Boltzmann methods for modeling microscale flow in fibrous porous media. Phys. Fluids 9(9), 24682474 (1997)

57. Tam, C.: The drag on a cloud of spherical particles in low Reynolds number flow. J. Fluid Mech. 38, 537-546 (1969)

58. Tan, H., Pillai, K.: Finite element implementation of stress-jump and stress continuity conditions at a porous medium clear fluid interface. Comput. Fluids 38(6), 1118-1131 (2009)

59. Vafai, K., Kim, S.: On the limitations of the Brinkman Forchheimer-extended Darcy equation. Int. J. Heat Fluid Flow 16(1), 11-15 (1995)

60. Valdes-Parada, F., Aguilar-Madera, C., Ochoa-Tapia, J., Goyeau, B.: Velocity and stress jump conditions between a porous medium and a fluid. Adv. Water Resour. 62, 327-339 (2013). doi:10.1016/j.advwatres.2013.08.008

61. Valdes-Parada, F., Alvarez-Ramirez, B., Goyeau, B., Ochoa-Tapia, J.: Computation of jump coefficients for momentum transfer between a porous medium and a fluid using a closed generalized transfer equation. Transp. Porous Media 78, 439-457 (2009)

62. Valdes-Parada, F., Goyeau, B., Ochoa-Tapia, J.: Jump momentum boundary condition at a fluid-porous dividing surface: derivation of the closure problem. Chem. Eng. Sci. 62, 4025-4039 (2007)

63. Whitaker, S.: Flow in porous media I: a theoretical derivation of Darcy's law. Transp. Porous Media 1, 3-25 (1986)

64. Whitaker, S.: The method of volume averaging, theory and applications of transport in porous media. Kluwer Academic, Dordrecht, The Netherlands (1999) 